\title{
3 Research Square

\section{Memory seeds enable high structural phase purity in 2D perovskite films for high-efficiency devices}

Siraj Sidhik

Rice University

Wenbin Li

Rice University

Mohammad H. K. Samani

Rice University https://orcid.org/0000-0001-9486-9978

Hao Zhang

Rice University

Yafei Wang

Rice University

Justin Hoffman

Northwestern University

Austin K. Fehr

Rice University

Michael Wong

Rice University https://orcid.org/0000-0002-3652-3378

Claudine Katan

Univ Rennes https://orcid.org/0000-0002-2017-5823

Jacky Even

Institut FOTON UMR CNRS 6082 https://orcid.org/0000-0002-4607-3390

Amanda B. Marciel

Rice University

Mercouri Kanatzidis

Northwestern University

Jean-Christophe Blancon

Rice University

Aditya Mohite ( $\nabla$ adm4@rice.edu )

Rice University https://orcid.org/0000-0001-8865-409X

Article 
Keywords: Halide Perovskites, Durable Optoelectronic Devices, Single-phase Crystalline Powders, Nucleation and Grain Growth

Posted Date: January 12th, 2021

DOI: https://doi.org/10.21203/rs.3.rs-90067/v1

License: (c) (1) This work is licensed under a Creative Commons Attribution 4.0 International License. Read Full License 


\title{
Memory seeds enable high structural phase purity in 2D perovskite films for high-efficiency devices
}

Siraj Sidhik ${ }^{1}$, Wenbin Li $^{1,2 \#}$, Mohammad H. K. Samani ${ }^{1 \# \text {, Hao Zhang }}{ }^{1,2}$, Yafei Wang ${ }^{1}$, Justin Hoffman ${ }^{3}$, Austin K. Fehr ${ }^{1}$, Michael Wong ${ }^{1}$, Claudine Katan ${ }^{4}$, Jacky Even ${ }^{5}$, Amanda B. Marciel ${ }^{1}$, Mercouri G. Kanatzidis ${ }^{3}$, Jean-Christophe Blancon ${ }^{1 *}$ and Aditya D. Mohite ${ }^{1^{*}}$

${ }^{1}$ Department of Chemical and Biomolecular Engineering, Rice University, Houston, Texas 77005, USA.

${ }^{2}$ Applied Physics Graduate Program, Smalley-Curl Institute, Rice University, Houston, Texas, 77005, USA.

${ }^{3}$ Department of Chemistry and Department of Materials Science and Engineering, Northwestern University, Evanston, Illinois 60208, USA.

${ }^{4}$ Univ Rennes, ENSCR, INSA Rennes, CNRS, ISCR (Institut des Sciences Chimiques de Rennes) - UMR 6226, F-35000 Rennes, France.

${ }^{5}$ Univ Rennes, INSA Rennes, CNRS, Institut FOTON, UMR 6082, Rennes F-35000, France

*Correspondence to: irb21@rice.edu, $\underline{\text { adm4@@rice.edu }}$

\# Authors with equal contribution

\begin{abstract}
Two-dimensional (2D) perovskites are a class of halide perovskites offering a pathway for realizing efficient and durable optoelectronic devices. However, the broad chemical phase space and lack of understanding of film formation have led to quasi-2D perovskite films with polydispersity in perovskite layer thicknesses, which have hindered devices performance and stability. Here, we demonstrate a scalable approach involving dissolution of single-phase crystalline powders with homogeneous perovskite layer thickness in desired solvents, to fabricate 2D perovskite thin-films with high phase purity. In-situ characterizations reveal the presence of sub-micron-sized seeds in solution that preserve the memory of the dissolved single-crystals and dictate the nucleation and growth of grains with identical thickness of the perovskite layers in thin-films. Photovoltaic devices fabricated with such films, yields an efficiency of $17.1 \%$ and $1.20 \mathrm{~V}$ open-circuit voltage, while preserving $97.5 \%$ of their peakperformance after 800 hours under illumination without any external thermal management.
\end{abstract}


Two-dimensional (2D) semiconductors exhibit extraordinary properties, which are relevant for a wide range of technologies such as optoelectronics, spintronics, and quantum information. ${ }^{1-3}$ However, the rich physics of these materials observed typically in high quality atomically-thin single crystals of micron-size has been elusive in scaling-up 2D material systems that have been integrated in devices. ${ }^{4,5}$ Recently, organic-inorganic $2 \mathrm{D}$ perovskites have emerged as a new family of 2D semiconductors with unique and tunable physical properties that can be preserved in bulk single crystals. ${ }^{6-14}$ Despite encouraging proof-of-concept thin-film devices, ${ }^{15-21}$ polydispersity or mixed crystal phases leads to a high density of structural phase impurities in films, ${ }^{16,22,23}$ which has limited device performance and prevented access to specific 2D physical properties in these films. ${ }^{10,14,24}$

The 2D perovskites synthetized in this work consist of nanometer-thick layers of organicinorganic halide perovskite with the formula $\mathrm{A}_{2}{ }_{2} \mathrm{~A}_{\mathrm{n}-1} \mathrm{M}_{\mathrm{n}} \mathrm{X}_{3 \mathrm{n}+1}$ (where $\mathrm{A}$ is a small organic cation, $\mathrm{M}$ is a metal, $\mathrm{X}$ is a halide, and $n$ defines the layer thickness) that are separated one from another by layers of large $\mathrm{A}^{\prime}$ organic cations. ${ }^{25,26} \mathrm{~A}$ key challenge is to design a reliable, generally applicable and scalable process to synthetize phase-pure 2D perovskite thin-films dominantly formed by a homogeneous distribution of a single perovskite layer thickness, and with a perovskite layer thickness intermediate between the $2 \mathrm{D}$ perovskite $n=1$ and the $3 \mathrm{D}$ perovskite limiting cases. ${ }^{24,27,28}$ However, the classical synthesis method typically yields a mixture of $n$-phase and 3 DPKs. ${ }^{23}$ There have been recent attempts to realize thin-films with narrow distribution of $n$-value 2D perovskites by using different solvents and organic additives that control the crystallization kinetics. Unfortunately, these efforts have had limited success, and do not appear to be applicable to all types of 2D perovskites. ${ }^{29-32}$ Our previously reported hot casting method seems to yield better phase purity in thin films on a few types of 2D perovskites, yet there is little understanding of the intermediate chemical steps in the formation of the $2 \mathrm{D}$ perovskite films. ${ }^{33} \mathrm{We}$ anticipate that the discovery of a reliable synthesis method to fabricate phase pure 2D perovskite thin films will enable optoelectronic devices that concomitantly exhibit high efficiency, tunability, scalability, and long-term operational stability, as well as pave the path for new types of devices, which exploit the rich physics of 2D materials. Indeed, studying films that are made of multiple phases frustrates our ability to understand the properties of the $2 \mathrm{D}$ perovskites. However, overcoming this longstanding and serious bottleneck mandates a deep understanding of the genesis of phase purity during thin-film formation, with the desired control over orientation and crystallinity. 
Here, we report on a phase-selective synthesis method involving a pre-crystallization step of 2D perovskite crystalline powders followed by single-step solution processing, to produce 2D perovskite thin-films principally formed from perovskite layers with a single-valued layer thickness (or $n$-value), with good crystallinity and desired orientation suitable for fabricating optoelectronic devices. Comprehensive in-situ X-ray diffraction and in-situ absorbance measurements with dynamic light scattering (DLS) analysis reveals the exact nature of 2D perovskite film formation where the growth is dominated by the nucleation of $200 \mathrm{~nm}$ homogeneous $n$-value seeds.

\section{Results and Discussion}

Thin film fabrication and structural characterization. The 2D perovskite thin films were fabricated using solution-processing methods at low temperature $\left(100^{\circ} \mathrm{C}\right.$ or less) following the two protocols described in Fig. 1a; a classical one-step protocol and our method termed as the "phaseselective" method. The classic synthesis method involves dissolving (by stirring for 12 hours) precursor materials (for example, $\mathrm{PbI}_{2}, \mathrm{BAI}$, and $\mathrm{MAI}$, with $\mathrm{BA}$ and $\mathrm{MA}$ standing for butylammonium and methylammonium, respectively) in stoichiometric proportions corresponding to the desired $n$-value in solution, followed by spin casting on a substrate and annealing at $100^{\circ} \mathrm{C} .{ }^{23}$ This approach yields thin-films composed of a polydisperse mixture of 2D perovskite $n$-phase materials. For example, when precursors are mixed in stoichiometric proportions to fabricate $\mathrm{BA}_{2} \mathrm{MA}_{2} \mathrm{~Pb}_{3} \mathrm{I}_{10}$ (i.e. Ruddlesden-Popper $n=3$ 2D perovskite), the classic synthesis method produces films composed of a mixture of the $n=2, n=3$, and $n=42 \mathrm{D}$ perovskite phases and often with evidences of the 3D halide perovskites. ${ }^{23,34}$ These films are often referred to as quasi-2D and denoted as $\langle n\rangle=3$ films. In order to promote phase selectivity during thin-film formation, we added an extra step in the synthesis protocol. First, we slowly crystallized 2D perovskite single crystal powders (micrometer to millimeter sizes) of a desired $n$-value from the precursor materials hereafter termed as parent-crystals (Fig. 1a). ${ }^{25,26}$ These parent crystals exhibited a high degree of phase purity, typically around 90 to $95 \%$ as validated using x-ray powder diffraction measurements. As illustrated in Fig. $1 \mathrm{~b}$ for the $\mathrm{BA}_{2} \mathrm{MA}_{2} \mathrm{~Pb}_{3} \mathrm{I}_{10}$ parent-crystal powders, the diffraction signal stems predominantly from the $n=3$ phase with less than $10 \%$ of $n=4$ impurities. The parent-crystals were then dissolved by heating at $70{ }^{\circ} \mathrm{C}$ in the optimized solvent for 6 or more hours and the resulting solution was spin casted and annealed. 


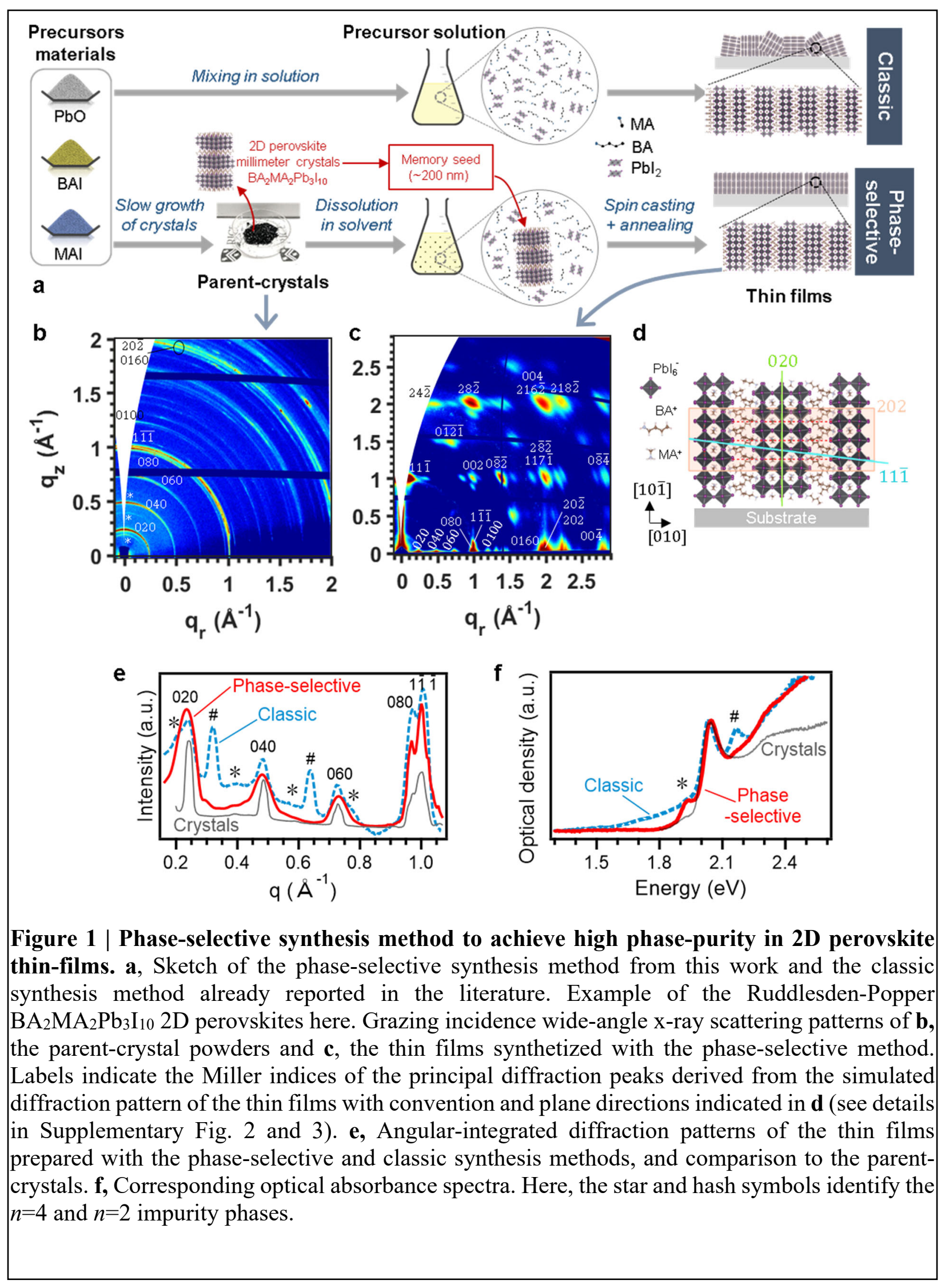


After optimization of the solvent composition (5\% MACl in DMF for the RuddlesdenPopper $\mathrm{BA}_{2} \mathrm{MA}_{2} \mathrm{~Pb}_{3} \mathrm{I}_{10}$ ) and dissolution process (Supplementary Fig. 1), we synthesized thinfilms, which exhibited a narrow variation of phase distribution in comparison to the classical method as verified by grazing incidence wide-angle x-ray scattering (GIWAXS) and absorption spectroscopy. The GIWAXS pattern of the $n=3$ Ruddlesden-Popper $2 \mathrm{D}$ perovskite films synthesized with the phase-selective method is shown in Fig. 1c. Its spot-like diffraction pattern indicates high crystallinity and preferential orientation of the perovskite layers as explained below. The experimental GIWAXS pattern was accurately reproduced and the diffraction peaks indexed from the $2 \mathrm{D}$ perovskite single crystal structure with vertically oriented perovskite layers (see the illustration of the structure in Fig. 1d and details of the analysis in the Supplementary Fig. 2 and 3). First, the simulated diffraction pattern and assignment of Miller indices to the experimental diffraction peaks yield a phase purity larger than $90 \%$ corresponding to a nearly homogeneous distribution of $\mathrm{BA}_{2} \mathrm{MA}_{2} \mathrm{~Pb}_{3} \mathrm{I}_{10}$ throughout the films even after the films were scratched into power form (see details in Supplementary Fig. 3). The degree of phase purity observed in oriented films was confirmed in crushed powders made by abrasing the thin films (Supplementary Fig. 4), which is opposite to the mixture of 2D perovskite phases with high $n$-value and 3D perovskites observed in crushed powders made from films prepared with the classis synthesis method. ${ }^{35}$ Moreover, we note that there are six equally spaced, low intensity peaks indicated by white circles in the GIWAXS pattern in Supplementary Fig. 3c,d that cannot be assigned to the normal reflections of the $n=32 \mathrm{D}$ perovskite phase or any other $n$-value phase, or to bulk halide perovskite. Based on the regular pattern formed by these peaks on the GIWAXS image, we infer that they correspond to the 003, 102, 203, and 104 Bragg reflections of the $\mathrm{BA}_{2} \mathrm{MA}_{2} \mathrm{~Pb}_{3} \mathrm{I}_{10}$ lattice that are normally forbidden by perfect single crystal space group symmetry (cell centering, glide planes and screw axis). Here, they can be observed due to local distortions of the perovskite octahedra and corrugation of the interface between the organic cation and perovskite layers. The diffraction integrated over all azimuthal directions of the GIWAXS pattern in Fig. 1e and the absorbance spectra of the thinfilms in Fig. 1f, both confirm a phase purity of about $90 \%$ for the $\mathrm{BA}_{2} \mathrm{MA}_{2} \mathrm{~Pb}_{3} \mathrm{I}_{10}$ films, which is largely determined by the degree of homogeneity in the perovskite layer thickness of the parentcrystals (Fig. 1b and gray curve in Fig. 1f). We point out here that the small values of absorbance below $1.8 \mathrm{eV}$ indicate a negligible amount of high $n$-value crystal phases or 3D-like perovskite in our films, and we attribute this small absorption to surface states and states resulting from the 
local distortions observed in the GIWAXS data. ${ }^{36-38}$ Secondly, using the Debye Scherrer analysis on the (111) diffraction peak (Supplementary Fig. 5), we extracted an average size of $31 \mathrm{~nm}$ for the grains forming the thin films. These values are comparable to those of the parent-crystals (average of $64 \mathrm{~nm}$ ), thus confirming that the crystalline quality is well maintained in the phaseselective thin films. Thirdly, the diffraction peaks of type $0 \mathrm{k} 0$ (with $\mathrm{k}=2 m$ and $m$ is integer) related to the layer stacking direction in the 2D perovskite crystals are observed predominantly along the qr-direction in the GIWAXS pattern in Fig. 1c ( $>90 \%$ based on the analysis in the Supplementary Fig. 2 and 3), indicating preferential out-of-plane orientation (normal to the substrate) of the perovskite layers as sketched in Fig. 1d. Finally, we analyzed the x-ray diffraction and absorption data of the thin-films fabricated with the classic synthesis method and determined that the films have significant polydispersity of perovskite layer thickness. We estimate a distribution of $n=3$ and $n=2$ phases in almost equal quantity, as well as the presence of $n=4$ and phases with higher $n$ value or 3D-like perovskite (blue dashed curve in Fig. 1e, f). To further confirm that our synthesis approach is applicable to $2 \mathrm{D}$ perovskite crystals with other compositions and structures, we performed measurements on the Dion Jacobson $n=32 \mathrm{D}$ perovskite thin films (4AMP-MA2 $\mathrm{Pb}_{3} \mathrm{I}_{10}$ where 4AMP is 4- Amino methyl piperidine, Supplementary Fig. 6, 7, and 8), which also demonstrated results consistent with those obtained for $n=3$ Ruddlesden-Popper thin-films.

Formation kinetics of the 2D perovskite thin films. The significant differences in the degree of phase purity and crystal orientation observed between the thin-films synthesized with the phaseselective and the classical method imply that the method of preparation of the solution before casting significantly affects the kinetics of formation of the 2D perovskite thin-films. For the classic synthesis method, Quintero-Bermudez et al. ${ }^{23}$ proposed a kinetic model showing that intermediate solvent complexes provide a framework for nucleation of the 2D perovskite layers during the annealing process. For the classic synthesis method, previous reports on trying to improve the phase purity in $2 \mathrm{D}$ perovskite thin films through control of the film formation kinetics, treatment of the solution to achieve smaller intermediate complexes, or change in chemical composition, have been limited to specific 2D perovskite composition and have often yielded mitigated success and reproducibility in terms of phase purity and quality of the thin films. ${ }^{39-41,30,31}$ In order to improve the reproducibility of thin-films with high phase purity and tailored composition, it is critical that we understand the differences in the crystallization kinetics and film formation between the two synthesis methods. Therefore, we performed an in-situ x-ray diffraction 
and absorbance of the thin-films during its growth, as illustrated in Fig. 2, which has shown to be a powerful approach to identify the different phases ( $n$ values) during the thin-film formation. ${ }^{23}$ These experiments were performed by spin casting the respective precursor solution on a glass microscope slide, which was kept at room temperature without annealing in order to slow down the kinetics of nucleation and film formation. We note that this process also yielded films like those obtained by post annealing (Supplementary Fig. 9). Fig. 2a and 2b illustrate the evolution of the diffraction pattern as a function of time from the moment the precursor solutions were spin casted up to eighty minutes of continuous monitoring of the $\mathrm{x}$-ray diffraction pattern. The signature diffraction peaks of each 2D perovskite phase were identified and monitored as a function of time with respect to the total diffraction of the sample (Fig. 2c, d). Both methods showed diffraction at about $\mathrm{q}=1 \AA^{-1}$ after one minute, indicative of the formation of the halide perovskite film, and after a few minutes most of the solution nucleated and formed 2D perovskite phases. We also observed a weak diffraction peak in the classic method around $0.57 \AA^{-1}$, which indicates the presence of intermediate phase complexes in the excess solution. ${ }^{23}$ Recent work on the classical synthesis method has demonstrated the existence of an intermediate gel phase during growth. ${ }^{42}$ This gel phase was identified as one of the processes that hinders the formation of homogenous $2 \mathrm{D}$ perovskite thin films. For film growths using the phase-selective method, the targeted $n=32 \mathrm{D}$ perovskite phase formed relatively more quickly and continued growing over time as the amount of excess solution is consumed (Fig. 2 a,c). Based on these data, we infer that the phase-selective synthesis method bypasses the intermediate phase (absence of diffraction peak corresponding to intermediate phase complex), which promotes the formation of the desired layer thickness with negligible phase segregation. In order to verify the different phases of thin-film formation, we also performed in-situ optical absorbance measurements as illustrated Fig. 2e, which corroborated that after a couple of minutes the solution nucleates dominantly into the $n=32 \mathrm{D}$ perovskite phase. The excess solution, which is not consumed initially, allows for further improvement of the purity of the $n=3$ phase at the expense of the $n=4$. The $n=4$ impurity phase decreased to a few percent after tens of minutes, as confirmed by both x-ray diffraction and absorbance data (Fig. 2a, c, e). On the other hand, analysis of the film formation in the classic synthesis method yields a mixture of $n=1$ and $n=2$ phases after a few minutes of nucleation (Fig. 2b, d, f). Subsequently, we observed the formation of the $n=32 \mathrm{D}$ perovskite phase over a period of tens of minutes, accompanied by the dissolution of the $n=12 \mathrm{D}$ perovskite crystals. The final film from the classic method at the end of 

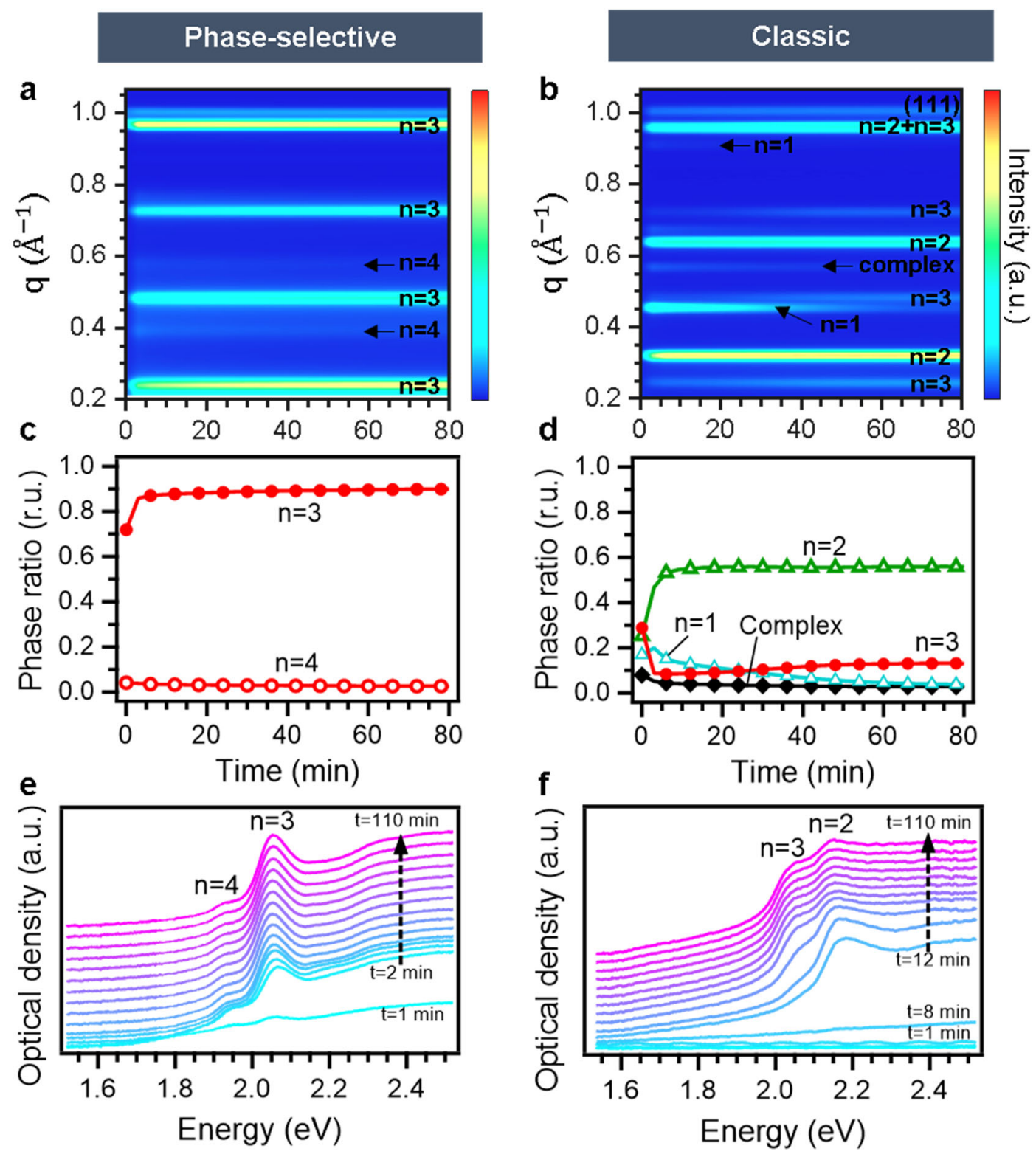

Figure 2 | In-situ structural and absorption characterization of the film formation in Ruddlesden-Popper $\mathbf{n}=\mathbf{3}$ perovskite. a, b, Evolution of the x-ray diffraction during film formation and comparison between our phase-selective synthesis method and the classic one. All peaks were identified to a $2 \mathrm{D}$ perovskite phase of defined $n$-value (or perovskite layer thickness), except for one peak assigned to the presence of an intermediate complex in solution. c, d, Corresponding evolution of the ratio of each phase relative to the fully integrated diffraction. e, f, Evolution of the optical absorbance spectra of the films during synthesis. Each peak in the spectra correspond to the ground exciton transition of a given perovskite layer thickness. 
our experiment was composed of a mixture of $n=2$ and $n=32 \mathrm{D}$ perovskites with little excess solution remaining. These in-situ experiments emphasize the important differences in film formation between the phase-selective method and the classic one. First, we note that in the phaseselective method, the desired 2D perovskites phase $(n=3)$ is readily formed as the dominant phase from the initial nucleation of the solution. In contrast, the classic method initially yields phases with lower $n$ value, followed by a subsequent slow growth of the expected phase. ${ }^{43,44}$ Second, the rate of nucleation is significantly faster in the phase-selective method compared to the classic approach, as validated from the absorbance data. These observations suggest that despite their apparently identical clear yellow colour and similar viscosity (Supplementary Fig. 10), there exist significant differences in the precursor solutions prepared for each synthesis method.

Direct visualization of $2 \mathrm{D}$ perovskite film growth and study of the precursor solution. In pursuit of a deeper understanding of the differences between the precursor solutions prepared using the two different synthesis methods, we performed in-situ microscopy to directly image the precursor solutions during the formation of 2D perovskite thin-films (Fig. 3a, b). We noticed striking differences in the nucleation and grain formation between the two synthesis protocols. In the case of the classic method, we observed a random nucleation of 2D perovskite grains with filament-like morphology, which grow to form the film (Fig. 3b). On the other hand, in the phaseselective method each grain nucleated at a single location and grew radially outward and independently from the other grains (Fig. 3a). These grain boundaries are formed either when two grains coalesced during the growth or when all reactants in solution are consumed and the growth terminates. The observation of nucleation and grain growth using the two protocols was captured in movies (Supplementary Movie 1 and Movie 2). The films synthesized with the phase-selective method featured relatively large (micron size) and ordered grains, whereas the classic method yielded disordered wire-like morphology (Fig. 3a, b, right side images, and confirmed by characterization of the surface morphology of post annealed films in Supplementary Fig. 11). We note that although we use the same time scale in Fig. $3 a$ and $3 b$, the resulting macroscopic growth of the films was in fact faster using the phase-selective method as observed during the in-situ experiments in Fig. 2. Here, we observe a large number of grains nucleated simultaneously in the phase-selective growth, whereas in the classic synthesis method, the film growth was slower and occurred at the interfaces between nucleated crystals and solution. These results support the 


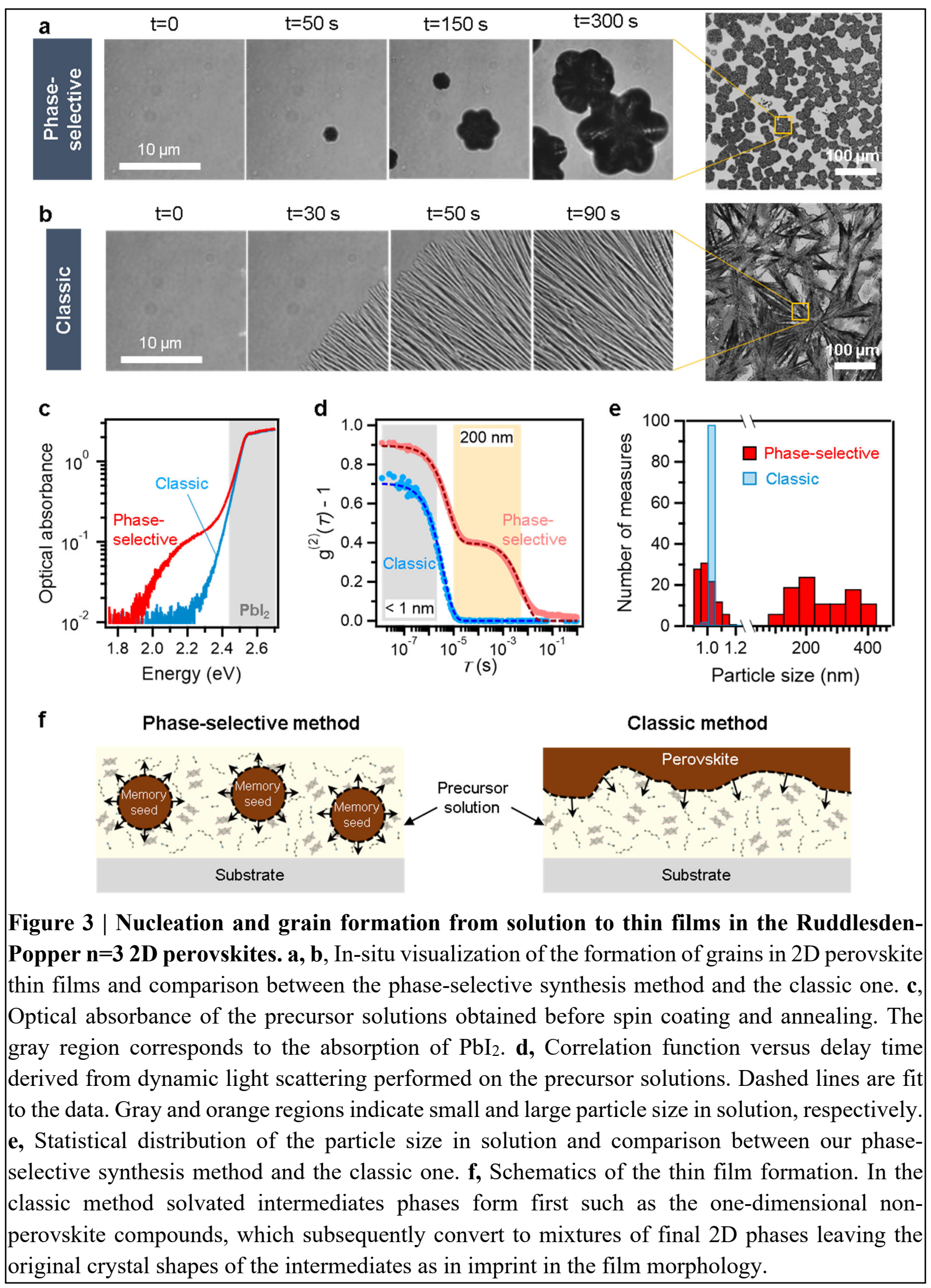


hypothesis that the precursor solutions in the phase-selective method contain a low-density of seeds for the nucleation of 2D perovskite grains as illustrated in the schematic of Fig. 1a. In addition, consistent with the analysis of the in-situ experiments in Fig. 2, we infer that the seeds in solution retain memory of the phase of the 2D perovskite parent-crystals, which were dissolved to form the precursor solution for film growth in the phase-selective synthesis method.

In order to determine the morphology and identity of these memory seeds, we performed dynamic light scattering and absorbance spectroscopy on the solutions before film casting (Fig. $3 \mathrm{c}-\mathrm{e})$. For the classic synthesis method, the optical absorbance spectra reflects the presence of $\mathrm{PbI}_{2}$ ions (absorbance below $2.4 \mathrm{eV}$ ), in agreement with the recent report of Quintero-Bermudez and co-workers. ${ }^{23}$ Using dynamic light scattering, we also confirmed that the typical size of these ions is approximately $1 \mathrm{~nm}$. On the other hand, the phase-selective method yields precursor solutions that in addition to the $\mathrm{PbI}_{2}$ absorbance spectrum have a finite absorbance between 2.0 and $2.4 \mathrm{eV}$. Moreover, the dynamic light scattering data indicate that in addition to the small ions $(\sim 1 \mathrm{~nm})$, there exists a much larger size distribution with an average particle size $\sim 200 \mathrm{~nm}$ (Fig. 3d, e and Supplementary Fig. 12). These results unambiguously confirm the presence of large-sized seeds in the precursor solution. The precursor solutions in both the phase-selective and classic methods are visually identical (Supplementary Fig. 10), and from the scaled down in-situ microscopic image shown in Fig. 3a, we estimate a concentration of less than $10^{-10} \mathrm{M}$ of active seeds in the precursor solution. Furthermore, a concentration-dependent dynamic light scattering measurement confirms the breakdown of the memory seeds into ions (Supplementary Fig. 13). By varying the dilution level (from $0.4 \mathrm{M}$ to $0.1 \mathrm{M}$ ) of $2 \mathrm{D}$ perovskite crystal solution, we found a critical point $(0.2$ M) at which the bimodal correlation (large seeds) changes to a unimodal correlation (ionic solution) and is attributed to the disruption of the chemical equilibrium between the memory seed and the solvent. Therefore, based on the dilution experiments and the absorbance data, it is reasonable to assume that the detected large seeds are indeed a remnant of the $n=3$ phase of the parent-crystals dissolved in solution. Since this step is absent for the classical synthesis method, we only measure the absorbance from $\mathrm{PbI}_{2}$ ions.

Figure $3 \mathrm{f}$ illustrates the mechanism of film formation in both types of synthesis methods. In the classic one the nucleation initiates at the air-liquid interface and the nucleation front sweeps across the film as discussed in previous work, ${ }^{45,46}$. In comparison, the phase-selective method the nucleation takes place preferentially at the memory seeds that create grains with high phase purity. 
We note that our approach of creating precursors by dissolving single-crystalline powers has also been adopted in fabricating films of 3D perovskites, which result in thin-films with better morphology and enhanced crystalline quality. ${ }^{47,48}$ More recently it was claimed that colloids identified as $\mathrm{MA}^{+} / \mathrm{PbI}_{\mathrm{x}}$ complexes promote the nucleation of mixed $n$-phase in $2 \mathrm{D}$ perovskite films. ${ }^{31}$ The needle-shaped crystal film morphology reflects the initial formation of solvated intermediates phases such as the one-dimensional non-perovskite $\left(\mathrm{CH}_{3} \mathrm{NH}_{3} \mathrm{PbI}_{3} \cdot \mathrm{DMF}\right)$ as has been recognized in $\mathrm{MAPbI}_{3}$ film growth previously. ${ }^{49}$ Then the final phase(s) form by subsequent reactions that accomplish the conversion. The phase-selective method avoids these solvated intermediates as the existing seeds act to grow the correct 2D phase. Indeed, our results are in sharp contrast to these past reports as we show that the seeds retain the memory of the parentcrystals, which upon nucleation lead to thin-films with high phase purity and crystallinity. It is apparent therefore that the two different methods have very different starting points and end up at very different destinations during the film formation process, with the phase-selective method affording purer single phase 2D films. Another advantage of the phase-selective method is that it facilitates a single step thin film deposition due to solvent independent seed assisted growth, thereby significantly improving film reproducibility critical for scaling-up in comparison to other deposition strategies like anti-solvent, hot casting, and sequential deposition. ${ }^{23,31,50}$ In addition, the concentration of the solvent can be tuned in the phase-selective method to achieve different size of the memory seeds and achieve solvent independent phase selective growth adapted to user's requirements (see Supplementary Fig. 1, 3 and 13).

Photovoltaic performances and devices stability. To illustrate the impact of phase purity of the $2 \mathrm{D}$ perovskite thin-films in devices, we fabricated $\mathrm{p}-\mathrm{i}-\mathrm{n}$ junction planar solar cells using the $2 \mathrm{D}$ perovskite films synthetized with the phase-selective method as absorbing layers. The device architecture used the standard ITO/HTL/2D-Perovskite/ETL/Al p-i-n architecture where PCBM and PEDOT:PSS served as the electron (ETL) and hole (HTL) transport layers, respectively, and is illustrated in Fig. 4a. Solar cells based on the Ruddlesden-Popper $\mathrm{BA}_{2} \mathrm{MA}_{2} \mathrm{~Pb}_{3} \mathrm{I}_{10}(\mathrm{n}=3)$ films prepared with the phase-selective method yielded peak power conversion efficiency of $12.60 \%$ with an open circuit voltage $\mathrm{V}_{\mathrm{OC}}=1.11 \mathrm{~V}$, short-circuit current density $\mathrm{J}_{\mathrm{SC}}=14.00 \mathrm{~mA} \cdot \mathrm{cm}^{-2}$, and fill factor of $83 \%$ (Fig. 4b). Similarly, the best solar cell devices using Dion Jacobson 4AMP$\mathrm{MA}_{2} \mathrm{~Pb}_{3} \mathrm{I}_{10}$ films exhibited comparable performances: an efficiency of about $11.50 \%$, Voc of 1.05 $\mathrm{V}$, and $\mathrm{J}_{\mathrm{SC}}$ of $14.06 \mathrm{~mA} \cdot \mathrm{cm}^{-2}$. For the photovoltaic devices with PEDOT:PSS as the HTL, we 
were unable to obtain a Voc exceeding $1.11 \mathrm{~V}$. To test if the solar cells were limited by the energy level alignment of the PEDOT:PSS with the 2D perovskite film valence band, and to optimize the power conversion efficiency, we replaced the PEDOT:PSS with NiOx as the HTL and fabricated devices using $\mathrm{BA}_{2} \mathrm{MA}_{2} \mathrm{~Pb}_{3} \mathrm{I}_{10}(n=3), \mathrm{BA}_{2} \mathrm{MA}_{3} \mathrm{~Pb}_{4} \mathrm{I}_{13}(n=4)$, and $4 \mathrm{AMP} \mathrm{MA}_{3} \mathrm{~Pb}_{4} \mathrm{I}_{13}(n=4)$ phase pure thin-films prepared with the phase-selective method as active layer (Fig. 4c). The currentvoltage characteristics of the optimized $\mathrm{BA}_{2} \mathrm{MA}_{2} \mathrm{~Pb}_{3} \mathrm{I}_{10}$ based device showed an increase in the overall efficiency from $12.60 \%$ to $14.3 \%$, arising from an increase in the Voc from $1.11 \mathrm{~V}$ to 1.22 $\mathrm{V}$. The champion device was obtained using a $\mathrm{BA}_{2} \mathrm{MA}_{3} \mathrm{~Pb}_{4} \mathrm{I}_{13}$ film prepared with the phase selective method, which showed a $\mathrm{J}_{\mathrm{SC}}=17.56 \mathrm{~mA} \cdot \mathrm{cm}^{-2}, \mathrm{~V}_{\mathrm{OC}}=1.20 \mathrm{~V}$, fill factor $81.1 \%$, and power conversion efficiency $17.1 \%$, with negligible hysteresis. The highest efficiency device using the Dion-Jacobson 4AMP-MA $\mathrm{Pb}_{4} \mathrm{I}_{13}$ film had a $\mathrm{J}_{\mathrm{SC}}=16.61 \mathrm{~mA} \mathrm{~cm}^{-2}$, $\mathrm{V}_{\mathrm{OC}}=1.11 \mathrm{~V}$, fill factor $83.0 \%$, and power conversion efficiency $15.7 \%$, with negligible hysteresis.

The statistics over tens of $\mathrm{BA}_{2} \mathrm{MA}_{3} \mathrm{~Pb}_{4} \mathrm{I}_{13}$ and $4 \mathrm{AMP}-\mathrm{MA}_{3} \mathrm{~Pb}_{4} \mathrm{I}_{13}$ devices presented in Fig. $4 \mathrm{~d}$ confirm the reproducibility of the solar cell performances and clearly demonstrate that the phase-selective method used to synthesize the 2D perovskite thin films yields large open-circuit voltage and fill-factor. The values obtained for the power conversion efficiency are among the best compared to previous results based on $<\mathrm{n}>$ quasi-2D perovskite solar cells. ${ }^{17,21,44,51-55}$ We note that although the use of quasi-2D perovskite films is beneficial for achieving current densities larger than $20 \mathrm{~mA} \cdot \mathrm{cm}^{-2}$ as compared to about $17 \mathrm{~mA} \cdot \mathrm{cm}^{-2}$ here with high phase purity films, it actually limits other figures of merit such open circuit voltage, fill factor, and stability in operation. The use of quasi-2D perovskite films indeed allows enhancing near-infrared absorption as compared to the lower values observed in high phase purity films (Fig. 1g and Supplementary Fig. 14) and achieves a balance between photoexcited electron-hole pair (or exciton) dissociation and material stability, however recent studies have demonstrated that a combination of the device field and lowdensity surface states can efficiently achieve exciton dissociation in 2D perovskite phases with low $n$-value. ${ }^{24,36,56} \mathrm{By}$ achieving high phase purity with the phase-selective method, 2D perovskite thin films naturally exhibit less structural defects, related to the polydispersity of classical films, which for example might result in stacking faults and dangling bonds between two adjacent layers of different thickness. ${ }^{23,34,37}$

The superior phase purity of our 2D perovskite films is expected to benefit device stability and to test this hypothesis, we performed stability measurements during operation of our 
$\mathrm{BA}_{2} \mathrm{MA}_{3} \mathrm{~Pb}_{4} \mathrm{I}_{13}$ solar cell devices. The cells were encapsulated and subjected to a constant 1-Sun illumination for up to 800 hours - under $60 \pm 5 \%$ relative humidity in air (Fig. 4e). The solar cells exhibit no "burn-in" effect ${ }^{57}$ and lost less than $3 \%$ of their initial performances over 800 hours as measured using a standard AM 1.5G source. On the contrary, our control solar cell prepared with

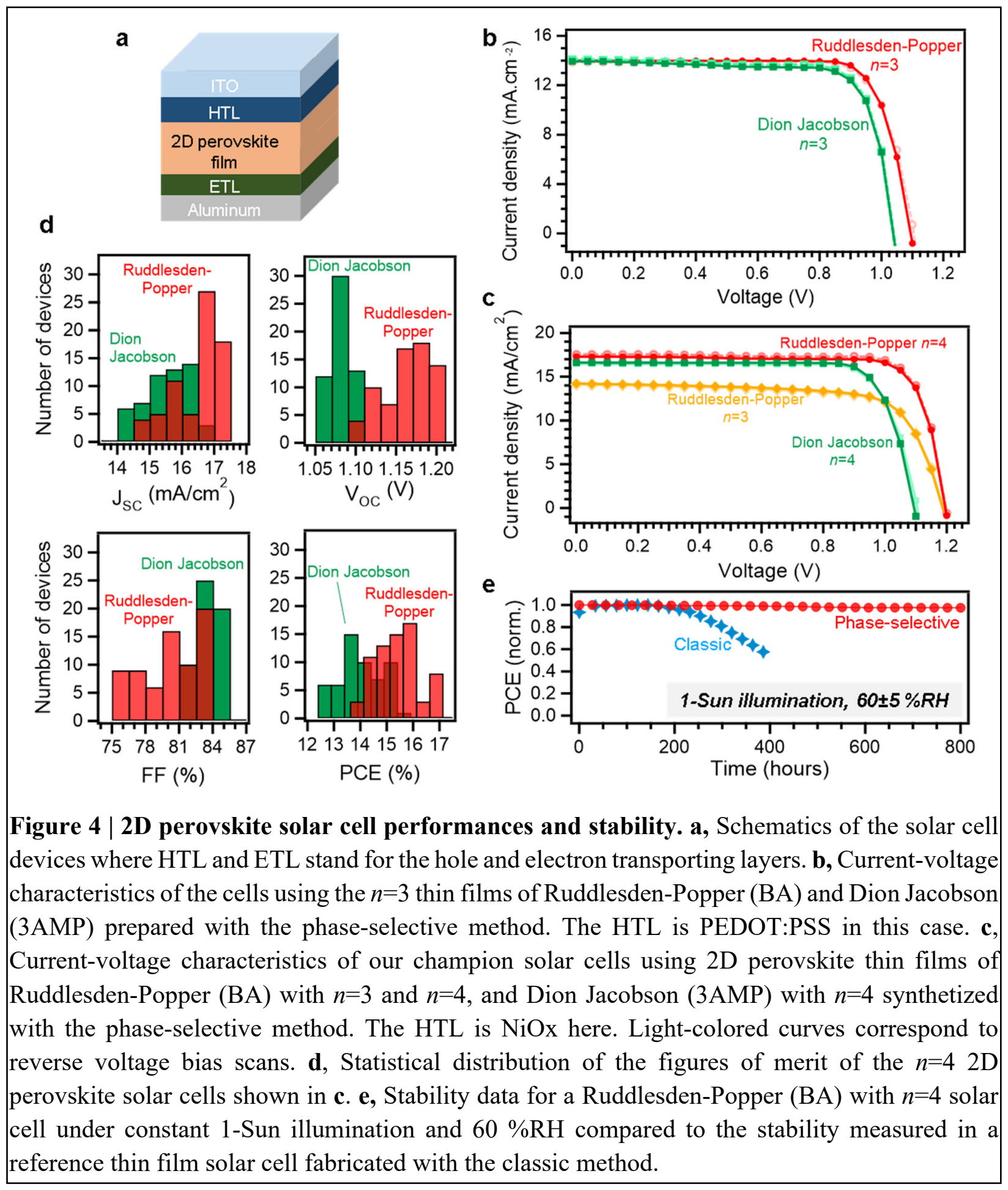


the classic synthesis method shows a burn-in effect and started to degrade after 200 hours. To the best of our knowledge, these stability results are better than state-of-art 2D perovskite devices with similar performances. ${ }^{21,51,53}$ These results pave a path for achieving dominantly phase pure thinfilms of 2D perovskites for realizing scalable, high-efficiency devices with long-term stability.

In summary, we developed a phase-selective synthesis method for fabricating 2D perovskite thin films with high phase purity, enhanced crystallinity and desired out-of-plane orientation. The in situ characterization indicates the absence of an intermediate phase complex and reveals a seed assisted nucleation and film growth process. The presence of these sub-micron-sized seeds in the solution which preserves the memory of the dissolved single-crystals was verified. The films fabricated by this method resulted in photovoltaic devices with an efficiency of $17.1 \%$, and enhanced stability.

\section{Methods}

2D perovskite parent crystal synthesis. The $2 \mathrm{D}$ perovskite parent crystals were synthesized by mixing $\mathrm{PbI}_{2}, \mathrm{MACl}$, and BAI (or 4AMPI in the case of the Dion Jacobson 2D perovskite) in appropriate ratios and dissolving these precursor materials in a mixture of $\mathrm{HI} / \mathrm{H}_{3} \mathrm{PO}_{2}$ according to

our previous reports..$^{25,26}$ The solution was stirred at high temperature $\left(>190^{\circ} \mathrm{C}\right)$ until all the precursors were dissolved, then allowed to cool down to room temperature during which time crystals are formed. The crystallized 2D perovskite powder was separated, dried, and characterized using x-ray diffraction and absorption.

2D perovskite thin film synthesis. For the classic method, the precursors, $\mathrm{BAI}, \mathrm{MAI}$, and $\mathrm{PbI}_{2}$ in appropriate ratios (with 5\% of $\mathrm{MACl}$ additive) were dissolved in N, N dimethylformamide (DMF). The solution was stirred overnight in an argon filled glove box. Then, the precursor solution was spin coated on the substrates and annealed at $100^{\circ} \mathrm{C}$ on a hot plate. In the phase-selective method, the parent crystals were dissolved in DMF with an addition of 5\% $\mathrm{MACl}$. After stirring the solution for at least $6 \mathrm{~h}$, it was spin casted on the substrates followed by annealing at $100^{\circ} \mathrm{C}$ on a hot plate. Detailed fabrication and solvent optimization techniques are presented in the method section of the Supplementary information.

In-situ X-Ray diffraction and absorption measurements. The precursor solutions were spin coated on glass substrates to obtain wet films and the samples were immediately transferred to the $\mathrm{x}$-ray diffraction apparatus for measurements. The area of the diffraction peaks were monitored 
over time until the $2 \mathrm{D}$ perovskite films were fully formed. The same approach was used to probe the evolution of the optical density of the films during formation.

GIWAXS measurement and analysis. The GIWAXS patterns were measured at the Advanced Photon Source (8-ID-E) and the National Synchrotron Light Source-II (11-BM). At the beamline 8-ID-E, the 2D perovskite parent crystals and thin film samples were placed on a Linkam grazing incidence $\mathrm{x}$-ray-scattering stage (temperature at $\left.25^{\circ} \mathrm{C}\right)$ inside a vacuum chamber $\left(10^{-4}\right.$ torr) with the sample $228 \mathrm{~mm}$ away from a Pilatus $1 \mathrm{M}$. The photon energy was $10.91 \mathrm{keV}$, and the beam size was $200 \mu \mathrm{m} \times 20 \mu \mathrm{m}(\mathrm{H} \mathrm{x} \mathrm{V})$. At the beamline 11-BM, the samples were measured inside a vacuum chamber $\left(10^{-2}\right.$ torr $)$ with the sample $267 \mathrm{~mm}$ away from a Pilatus $800 \mathrm{~K}$. The photon energy was $13.5 \mathrm{keV}$, and the beam size was $200 \mu \mathrm{m}$ x $50 \mu \mathrm{m}(\mathrm{H} \mathrm{x} \mathrm{V})$. All GIWAXS patterns were taken at $0.15^{\circ}$ incident angle with typically 5 second exposure. The GIWAXS patterns were analyzed using the GIXSGUI package and Single Crystal-Crystal Maker software. ${ }^{58}$

In-situ microscopic imaging. Few microliters of the precursor solutions were drop casted on a thin microscopic slide that was then placed in a Titan microscope. The thin film formation without annealing was imaged using an oil immersion $100 \mathrm{X}$ objective over time by taking continuous snapshot images every second.

Dynamic light scattering measurements. The precursor solutions were loaded into cylindrical glass cuvettes and the DLS correlation curves were collected at different angle with respect to the incident laser beam. The data were fitted using a single- or bi-exponential decay model to extract the size of the particles in the solution (see details in the method section of the Supplementary information).

Solar cell device fabrication. ITO glasses were cleaned by ultra-sonication in soap water, distilled water, acetone, acetone/ethanol (50:50) and isopropyl alcohol for $15 \mathrm{~min}$ each, followed by UV treatment for 30 minutes. A thin layer of PEDOT: PSS or $\mathrm{NiO}_{x}$ was spin-coated on the ITO substrates at 5,000 rpm for 30 seconds to form the hole-transporting layer. The coated substrates were then transferred to an argon-filled glovebox where the $2 \mathrm{D}$ perovskite thin films were synthetized by spin coating and annealing at $100^{\circ} \mathrm{C}$ for $10 \mathrm{~min}$. The assemblies were completed by spin-coating (at 1,000 rpm for 300 seconds) a thin layer of PCBM (electron-transporting layer) and depositing on top aluminium contacts using a thermal evaporator with a shadow mask having a working area of $31.4 \mathrm{~mm}^{2}$. 
Solar cell Characterization. The solar cells were characterized by measuring the current-voltage curves while the cells are exposed to the light from a Newport ABB solar simulator (AM 1.5 G light). The external quantum efficiencies were collected by illuminating the device using the monochromatic light obtained from a quartz-tungsten-halogen source modulated at $2 \mathrm{kHz}$. The obtained photocurrent was measured by a lock-in amplifier. For stability measurements, the perovskite devices were encapsulated with a UV- curable epoxy and tested under open-circuit conditions, under full-spectrum simulated AM $1.5 \mathrm{G}$ in air using the same ABB solar simulator (following the ISOS-L protocol). The relative humidity was measured to be constant at $60 \pm 5 \% \mathrm{RH}$.

Acknowledgments: The work at Rice University was supported by the DOE-EERE 2022-1652 program. J.E. acknowledges the financial support from the Institut Universitaire de France. This work was supported by the Office of Naval Research (ONR) under grant N00014-20-1-2725.. This research used facilities of the Advanced Photon Source, a U.S Department of Energy (DOE) Office of Science User Facility operated for the DOE Office of Science by Argonne National Laboratory under Contrast No. DE-AC02-06CH11357. This research used the beamline 11-BM of the National Synchrotron Light Source II, a U.S. Department of Energy (DOE) Office of Science user facility operated for the DOE Office of Science by Brookhaven National Laboratory under Contract No. DE-SC0012704.

Author contributions: J.-C.B. and A.D.M. developed the concept, designed the experiment, and wrote the manuscript. S.S. synthesized the perovskite crystals in collaboration with J.H. under the supervision of M.G.K. S.S. also fabricated thin films, characterized the materials, performed insitu x-ray diffraction and absorbance, fabricated the solar cells, and performed solar cell characterization with support from Y.W., H.Z.. W.L. measured and analyzed the GIWAXS patterns with the help of A.K.F. and performed indexing with guidance from J.E. and C.K. Under A.B.M's supervision, M.S. performed the dynamic light scattering measurements and analyzed the size distribution; he worked with S.S. on in-situ imaging of the film formation. All authors read the manuscript and agree to its contents, and all data are reported in the main text and supplemental materials.

Competing interests: The authors declare no competing interests. 


\section{References}

1. Novoselov, K. S., Mishchenko, A., Carvalho, A. \& Neto, A. H. C. 2D materials and van der Waals heterostructures. Science 353, aac9439 (2016).

2. Schaibley, J. R. et al. Valleytronics in 2D materials. Nat. Rev. Mater. 1, 1-15 (2016).

3. Liu, X. \& Hersam, M. C. 2D materials for quantum information science. Nat. Rev. Mater. 4, 669-684 (2019).

4. Rhodes, D., Chae, S. H., Ribeiro-Palau, R. \& Hone, J. Disorder in van der Waals heterostructures of 2D materials. Nat. Mater. 18, 541-549 (2019).

5. Moving towards the market. Nat. Mater. 18, 519-519 (2019).

6. Ishihara, T., Takahashi, J. \& Goto, T. Optical properties due to electronic transitions in twodimensional semiconductors $\left(\mathrm{C}_{\mathrm{n}} \mathrm{H}_{2 \mathrm{n}+1} \mathrm{NH}_{3}\right)_{2} \mathrm{PbI}_{4}$. Phys. Rev. B 42, 11099-11107 (1990).

7. Mitzi, D. B., Feild, C. A., Harrison, W. T. A. \& Guloy, A. M. Conducting tin halides with a layered organic-based perovskite structure. Nature 369, 467-469 (1994).

8. Papavassiliou, G. C. \& Koutselas, I. B. Structural, optical and related properties of some natural three- and lower-dimensional semiconductor systems. Synth. Met. 71, 1713-1714 (1995).

9. Saparov, B. \& Mitzi, D. B. Organic-Inorganic Perovskites: Structural Versatility for Functional Materials Design. Chem. Rev. 116, 4558-4596 (2016).

10. Mao, L., Stoumpos, C. C. \& Kanatzidis, M. G. Two-dimensional hybrid halide perovskites: principles and promises. J. Am. Chem. Soc. 141, 1171-1190 (2019).

11. Blancon, J.-C. et al. Scaling law for excitons in 2D perovskite quantum wells. Nat. Commun. 9, 2254 (2018).

12. Gong, X. et al. Electron-phonon interaction in efficient perovskite blue emitters. Nat. Mater. 17, 550-556 (2018).

13. Long, G. et al. Chiral-perovskite optoelectronics. Nat. Rev. Mater. 5, 423-439 (2020).

14. Leng, K., Fu, W., Liu, Y., Chhowalla, M. \& Loh, K. P. From bulk to molecularly thin hybrid perovskites. Nat. Rev. Mater. 1-19 (2020) doi:10.1038/s41578-020-0185-1.

15. Kagan, C. R., Mitzi, D. B. \& Dimitrakopoulos, C. D. Organic-inorganic hybrid materials as semiconducting channels in thin-film field-effect transistors. Science 286, 945-947 (1999).

16. Yuan, M. et al. Perovskite energy funnels for efficient light-emitting diodes. Nat. Nanotechnol. 11, 872-877 (2016).

17. Tsai, H. et al. High-efficiency two-dimensional Ruddlesden-Popper perovskite solar cells. Nature 536, 312 (2016).

18. Wang, Z. et al. Efficient ambient-air-stable solar cells with 2D-3D heterostructured butylammonium-caesium-formamidinium lead halide perovskites. Nat. Energy 2, 1-10 (2017). 
19. Zhao, B. et al. High-efficiency perovskite-polymer bulk heterostructure light-emitting diodes. Nat. Photonics 12, 783-789 (2018).

20. Smith, M. D. \& Karunadasa, H. I. White-light emission from layered halide perovskites. Acc. Chem. Res. 51, 619-627 (2018).

21. Luo, T. et al. Compositional control in 2D perovskites with alternating cations in the interlayer space for photovoltaics with efficiency over 18\%. Adv. Mater. 31, 1903848 (2019).

22. Shang, Q. et al. Unveiling structurally engineered carrier dynamics in hybrid quasi-twodimensional perovskite thin films toward controllable emission. J. Phys. Chem. Lett. 8, 44314438 (2017).

23. Quintero-Bermudez, R. et al. Compositional and orientational control in metal halide perovskites of reduced dimensionality. Nat. Mater. 17, 900 (2018).

24. Grancini, G. \& Nazeeruddin, M. K. Dimensional tailoring of hybrid perovskites for photovoltaics. Nat. Rev. Mater. 4, 4-22 (2019).

25. Stoumpos, C. C. et al. Ruddlesden-Popper hybrid lead iodide perovskite 2D homologous semiconductors. Chem. Mater. 28, 2852-2867 (2016).

26. Mao, L. et al. Hybrid Dion-Jacobson 2D lead iodide perovskites. J. Am. Chem. Soc. 140, 3775-3783 (2018).

27. Krishna, A., Gottis, S., Nazeeruddin, M. K. \& Sauvage, F. Mixed dimensional 2D/3D hybrid perovskite absorbers: the future of perovskite solar cells? Adv. Funct. Mater. 29, 1806482 (2019).

28. Huang, P., Kazim, S., Wang, M. \& Ahmad, S. Toward Phase Stability: Dion-Jacobson Layered Perovskite for Solar Cells. ACS Energy Lett. 4, 2960-2974 (2019).

29. Wang, Y.-K. et al. Chelating-agent-assisted control of $\mathrm{Cs}_{\mathrm{PbBr}}$ quantum well growth enables stable blue perovskite emitters. Nat. Commun. 11, 3674 (2020).

30. Zhou, M., Fei, C., Sarmiento, J. S. \& Wang, H. Manipulating the phase distributions and carrier transfers in hybrid quasi-two-dimensional perovskite films. Sol. RRL 3, 1800359 (2019).

31. He, T. et al. Reduced-dimensional perovskite photovoltaics with homogeneous energy landscape. Nat. Commun. 11, 1672 (2020).

32. Han, Y., Park, S., Kim, C., Lee, M. \& Hwang, I. Phase control of quasi-2D perovskites and improved light-emitting performance by excess organic cations and nanoparticle intercalation. Nanoscale 11, 3546-3556 (2019).

33. Tsai, H. et al. High-efficiency two-dimensional Ruddlesden-Popper perovskite solar cells. Nature 536, 312-316 (2016).

34. Venkatesan, N. R. et al. Phase intergrowth and structural defects in organic metal halide Ruddlesden-Popper thin films. Chem. Mater. 30, 8615-8623 (2018).

35. Lin, Y. et al. Unveiling the operation mechanism of layered perovskite solar cells. Nat. Commun. 10, 1008 (2019). 
36. Blancon, J.-C. et al. Extremely efficient internal exciton dissociation through edge states in layered 2D perovskites. Science 355, 1288-1292 (2017).

37. Kepenekian, M. et al. Concept of lattice mismatch and emergence of surface states in twodimensional hybrid perovskite quantum wells. Nano Lett. 18, 5603-5609 (2018).

38. Raja, A. et al. Dielectric disorder in two-dimensional materials. Nat. Nanotechnol. 14, 832837 (2019).

39. Liu, J., Leng, J., Wu, K., Zhang, J. \& Jin, S. Observation of Internal Photoinduced Electron and Hole Separation in Hybrid Two-Dimentional Perovskite Films. J. Am. Chem. Soc. 139, 1432-1435 (2017).

40. Zheng, K. et al. Inter-phase charge and energy transfer in Ruddlesden-Popper 2D perovskites: critical role of the spacing cations. J. Mater. Chem. A 6, 6244-6250 (2018).

41. Cheng, P. et al. Highly Efficient Ruddlesden-Popper Halide Perovskite PA2MA4Pb5I16 Solar Cells. ACS Energy Lett. 3, 1975-1982 (2018).

42. Liu, L. et al. Cation Diffusion Guides Hybrid Halide Perovskite Crystallization during the Gel Stage. Angew. Chem. Int. Ed. 59, 5979-5987 (2020).

43. Soe, C. M. M. et al. Structural and thermodynamic limits of layer thickness in 2D halide perovskites. Proc. Natl. Acad. Sci. 116, 58-66 (2019).

44. Quan, L. N. et al. Ligand-Stabilized Reduced-Dimensionality Perovskites. J. Am. Chem. Soc. 138, 2649-2655 (2016).

45. Chen, A. Z. et al. Origin of vertical orientation in two-dimensional metal halide perovskites and its effect on photovoltaic performance. Nat. Commun. 9, 1336 (2018).

46. Dong, J. et al. Mechanism of Crystal Formation in Ruddlesden-Popper Sn-Based Perovskites. Adv. Funct. Mater. 30, 2001294 (2020).

47. Zhao, Y. et al. Perovskite seeding growth of formamidinium-lead-iodide-based perovskites for efficient and stable solar cells. Nat. Commun. 9, 1607 (2018).

48. Yen, H.-J. et al. Large Grained Perovskite Solar Cells Derived from Single-Crystal Perovskite Powders with Enhanced Ambient Stability. ACS Appl. Mater. Interfaces 8, 14513-14520 (2016).

49. Hao, F., Stoumpos, C. C., Liu, Z., Chang, R. P. H. \& Kanatzidis, M. G. Controllable Perovskite Crystallization at a Gas-Solid Interface for Hole Conductor-Free Solar Cells with Steady Power Conversion Efficiency over 10\%. J. Am. Chem. Soc. 136, 16411-16419 (2014).

50. Zhang, X. et al. Stable high efficiency two-dimensional perovskite solar cells via cesium doping. Energy Environ. Sci. 10, 2095-2102 (2017).

51. Cohen, B.-E., Li, Y., Meng, Q. \& Etgar, L. Dion-Jacobson Two-Dimensional Perovskite Solar Cells Based on Benzene Dimethanammonium Cation. Nano Lett. 19, 2588-2597 (2019). 
52. Li, H. et al. Layered Ruddlesden-Popper Efficient Perovskite Solar Cells with Controlled Quantum and Dielectric Confinement Introduced via Doping. Adv. Funct. Mater. 29, 1903293 (2019).

53. Niu, T. et al. Reduced-dimensional perovskite enabled by organic diamine for efficient photovoltaics. J. Phys. Chem. Lett. 10, 2349-2356 (2019).

54. Zheng, Y. et al. Oriented and Uniform Distribution of Dion-Jacobson Phase Perovskites Controlled by Quantum Well Barrier Thickness. Sol. RRL 3, 1900090 (2019).

55. Zhang, F. et al. Advances in two-dimensional organic-inorganic hybrid perovskites. Energy Environ. Sci. 13, 1154-1186 (2020).

56. Tsai, H. et al. Design principles for electronic charge transport in solution-processed vertically stacked 2D perovskite quantum wells. Nat. Commun. 9, (2018).

57. Khenkin, M. V. et al. Consensus statement for stability assessment and reporting for perovskite photovoltaics based on ISOS procedures. Nat. Energy 5, 35-49 (2020).

58. Jiang, Z. et al. The dedicated high-resolution grazing-incidence X-ray scattering beamline 8ID-E at the Advanced Photon Source. J. Synchrotron Radiat. 19, 627-636 (2012). 


\section{Supplementary Information for}

\section{Memory seeds enable high structural phase purity in 2D perovskite films for high-efficiency devices}

Siraj Sidhik ${ }^{1}$, Wenbin $\mathrm{Li}^{1,2 \#}$, Mohammad H. K. Samani ${ }^{1 \#}$, Hao Zhang ${ }^{1,2}$, Yafei Wang ${ }^{1}$, Justin Hoffman ${ }^{3}$, Austin K. Fehr ${ }^{1}$, Michael Wong ${ }^{1}$, Claudine Katan ${ }^{4}$, Jacky Even ${ }^{5}$, Amanda B. Marciel ${ }^{1}$, Mercouri G. Kanatzidis ${ }^{3}$, Jean-Christophe Blancon ${ }^{1 *}$ and Aditya D. Mohite ${ }^{1 *}$

${ }^{1}$ Department of Chemical and Biomolecular Engineering, Rice University, Houston, Texas 77005, USA.

${ }^{2}$ Applied Physics Graduate Program, Smalley-Curl Institute, Rice University, Houston, Texas, 77005, USA.

${ }^{3}$ Department of Chemistry and Department of Materials Science and Engineering, Northwestern University, Evanston, Illinois 60208, USA.

${ }^{4}$ Univ Rennes, ENSCR, INSA Rennes, CNRS, ISCR (Institut des Sciences Chimiques de Rennes) - UMR 6226, F-35000 Rennes, France.

${ }^{5}$ Univ Rennes, INSA Rennes, CNRS, Institut FOTON, UMR 6082, Rennes F-35000, France

*Correspondence to: irb21@rice.edu, $\underline{\text { adm4@rice.edu }}$ 


\section{Methods and Characterization}

\subsection{Thin films synthesis}

Classic synthesis method: The precursor solution of two-dimensional (2D) Ruddlesden popper perovskite $\mathrm{BA}_{2} \mathrm{MA}_{2} \mathrm{~Pb}_{3} \mathrm{I}_{10}$ was prepared by mixing lead Iodide $\left(\mathrm{PbI}_{2}\right.$, Sigma Aldrich, Perovskite grade), methylammonium iodide (MAI, GreatCell Solar, $\geq 99.9 \%$ ), and n-butylammonium iodide (BAI, Sigma Aldrich, $\geq 98 \%$ ) in an appropriate stoichiometry $(3: 2: 2)$ to attain a concentration of 0.5-1 M in N,N-dimethylformamide (DMF, Sigma Aldrich, $\geq 99.9 \%$, anhydrous). A 5\% of methylammonium chloride ( $\mathrm{MACl}$, Lumtec, $\geq 99.9 \%$ ) with respect to the weight of $\mathrm{PbI}_{2}$ was introduced as an additive in the solution. Further, the prepared solution was left stirring at $70^{\circ} \mathrm{C}$ for overnight inside an argon-filled glovebox. After cooling down, $100 \mu \mathrm{l}$ of the prepared solution was dropped at the center of a 1'x1' substrate and spin coated at $4000 \mathrm{rpm}$ for $30 \mathrm{sec}$ followed by annealing at $100^{\circ} \mathrm{C}$ for $10 \mathrm{~min}$. All spin coating was performed in an inert atmosphere.

Phase-selective synthesis method: This synthesis approach comprises the two steps described below.

Step 1: Parent-crystal powder synthesis: Following our previously reported method, ${ }^{25}$ the $2 \mathrm{D}$ Ruddlesden popper perovskite parent-crystals of $\mathrm{BA}_{2} \mathrm{MA}_{2} \mathrm{~Pb}_{3} \mathrm{I}_{10}$ were synthesized by combining lead oxide ( $\mathrm{PbO}$, Sigma Aldrich, 99\%), methylamine hydrochloride ( $\mathrm{MACl}$, Sigma Aldrich, $\geq 98 \%$ ) and butylamine (BA, Sigma Aldrich, 99.5\%) in appropriate ratios in a mixture of hydroiodic acid ( $\mathrm{HI}, 57 \mathrm{wt} \%$ in $\left.\mathrm{H}_{2} \mathrm{O}\right)$ and hypophosphorous acid $\left(\mathrm{H}_{3} \mathrm{PO}_{2}, 50 \%\right.$ in $\left.\mathrm{H}_{2} \mathrm{O}\right)$. The solution was stirred at a temperature of $190^{\circ} \mathrm{C}$ until all the precursors materials were dissolved, and the solution starts boiling. Then, the precursor solution was left to cool down to room temperature that led to the crystallization process of flat single crystals with micrometer to millimeter characteristic size. The quality and phase purity of each batch of parent-crystals synthesized were characterized by a combination of x-ray diffraction and absorbance measurements.

The Dion Jacobson perovskite parent-crystals of (4AMP) $\mathrm{MA}_{2} \mathrm{~Pb}_{3} \mathrm{I}_{10}$ were prepared by combining $\mathrm{PbO}, \mathrm{MAI}$, and 4- Aminomethyl piperidine (4AMP, Sigma Aldrich, 96\%) in appropriate ratios in a $\mathrm{HI} / \mathrm{H}_{3} \mathrm{PO}_{2}$ solvent mixture. The solution was stirred at a high temperature of $240^{\circ} \mathrm{C}$ until all the precursors were dissolved, and the solution starts boiling. Then, the solution was left to cool down to room temperature that led to the crystallization process. A detailed synthesis procedure is reported in ref. ${ }^{26}$.

Step 2: Solution processing and film fabrication: To fabricate the thin films, we prepared the precursor solution by dissolving the $2 \mathrm{D}$ perovskite parent-crystal powders synthesized above into a solvent solution (see below for details about the different types of solvent tested). The prepared solution was left on the hotplate at $70^{\circ} \mathrm{C}$ for $6 \mathrm{~h}$. After cooling down, $100 \mu \mathrm{l}$ of the prepared solution was instantly dropped and spin coated over substrates at 4000 r.p.m for 30 s followed by heating at $100^{\circ} \mathrm{C}$ for $10 \mathrm{~min}$ resulting in the formation of the $2 \mathrm{D}$ perovskite thin films.

In order to achieve thin films with the best phase purity and desired crystal orientation, we tested a range of solvent in which the $2 \mathrm{D}$ perovskite parent-crystals were dissolved:

- DMF $=0.4 \mathrm{~g}$ of parent-crystals in $1 \mathrm{ml}$ of anhydrous DMF.

- DMSO $=0.4 \mathrm{~g}$ of parent-crystals in $1 \mathrm{ml}$ of anhydrous DMSO. 
- DMF:DMSO = $0.4 \mathrm{~g}$ of parent-crystals in $1 \mathrm{ml}$ of anhydrous DMF/DMSO (1:1).

- DMF:DMSO-HI = DMSO: $0.4 \mathrm{~g}$ of parent-crystals in $1 \mathrm{ml}$ of anhydrous DMF/DMSO (1:1) with $1 \mu 1$ of HI.

- DMF-MACl $=0.4 \mathrm{~g}$ of parent-crystals and $0.02 \mathrm{~g}$ of dried MACl in $1 \mathrm{ml}$ of anhydrous DMF, similar to the method reported in ref. ${ }^{21}$.

\subsection{Solar cell devices fabrication}

We used an inverted planar architecture ITO/HTL/2D-perovskite-film/ETL/Al for the solar cells as depicted by the schematic diagram in Fig. 4a, and fabricated two types of devices to achieve the best performances. One type of solar cell combines PEDOT:PSS (Clevios ${ }^{\mathrm{TM}}$ PEDOT:PSS, Heraeus Epurio) as the HTL (hole transporting layer) and [6,6]-phenyl-C61-butyric acid methyl ester (PCBM, Sigma Aldrich, 99.9\%) as the ETL (electron transport layer). In the second case, nickel oxide $\left(\mathrm{NiO}_{\mathrm{x}}\right)$ was used as the HTL and PCBM as the ETL with a $\sim 1 \mathrm{~nm}$ passivating layer of bathocuproine (BCP, Sigma Aldrich, 96\%).

Here we detail the device fabrication procedure. First, we prepared the top part of our solar cells consisting of the patterned indium doped tin oxide (ITO, Thin Film Device Inc.) substrates and the hole transporting layer (HTL). The ITO substrates were washed in water, acetone, acetone/ethanol (50:50) and isopropyl alcohol by ultrasonication for $15 \mathrm{~min}$, respectively. The substrates were further dried under argon airflow and treated with UV for $30 \mathrm{~min}$. Then, a PEDOT:PSS or $\mathrm{NiO}_{\mathrm{x}}$ layer was spin-coated on the clean ITO substrates at 5000 revolutions per minute (r.p.m.) for $30 \mathrm{~s}$ (layer thickness was about $30 \mathrm{~nm}$ ). The $\mathrm{NiOx}$ solution was prepared by dissolving Nickel(II) acetate tetrahydrate (Sigma Aldrich, 99.995\%) in absolute ethanol (Sigma Aldrich, 200 proof) with monoethanolamine (MEA, Sigma Aldrich, $\geq 99 \%$ ). The ratio of $\mathrm{Ni}^{2+}$ and MEA was chosen to be $1: 1$. The solution was stirred at $60^{\circ} \mathrm{C}$ for 1 hour until a clear and deep green solution was obtained. The ITO/PEDOT:PSS or NiOx substrates were subsequently transferred to an argon-filled glovebox for the rest of the solar cell fabrication process.

In the glovebox, the 2D perovskite thin films with about $250 \mathrm{~nm}$ thickness was fabricated onto the ITO/PEDOT:PSS substrates followed by deposition of the electron transport layer, PCBM. The PCBM solution was prepared by dissolving $25 \mathrm{mg}$ PCBM in $1 \mathrm{ml}$ of chlorobenzene, followed by overnight stirring at $60^{\circ} \mathrm{C}$. A $50 \mu 1$ of the PCBM solution was dropped on the ITO/PEDOT/2D-Perovskite samples and spin-coated at 1000 r.p.m. for $45 \mathrm{~s}$ to form a thin layer of $\sim 30 \mathrm{~nm}$. The solar cell devices were completed by evaporating a $100 \mathrm{~nm}$ layer of aluminum using a shadow mask yielding eight cells of $31.4 \mathrm{~mm}^{2}$ per 1'x1' area of the sample device.

\subsection{X-ray diffraction and in-situ $\mathrm{x}$-ray diffraction measurements}

$X$-ray diffraction of crystals: Batches of synthesized 2D perovskite crystals were characterized using one-dimensional X-Ray diffraction. The synthesized parent-crystal powders were extracted immediately after the crystallization process to be dried using a Whatmann filter paper. The measurement was carried out in the q-range between 0.2 and $1.6 \AA^{-1}$ corresponding to $2 \theta$ between $3^{\circ}$ and $15^{\circ}$ with a step of $0.01^{\circ}$ and a speed of 2 degrees per minute using a Rigaku SmartLab X- 
Ray diffractometer with $\mathrm{Cu}(\mathrm{K} \boldsymbol{\alpha})$ radiation $(\lambda=1.5406 \AA)$. The corresponding Bragg peaks were identified and compared with the simulated diffraction obtained from the $2 \mathrm{D}$ perovskite structures discovered previously. ${ }^{25,26}$ In case of the presence of impurities or mixed phases, the synthesized crystals were discarded, and the experiment was repeated until obtaining a parent-crystal powder with high phase purity (i.e. $>90 \%$ of a single $n$-value).

$X$-ray diffraction of thin films: The $\mathrm{x}$-ray diffraction of the thin films was measured in the q-range between 0.2 and $2.25 \AA^{-1}$ corresponding to $2 \theta$ between $3^{\circ}$ and $32^{\circ}$, with a step of $0.01^{\circ}$ and a speed of 2 degrees per minute, using a Rigaku SmartLab X-Ray diffractometer with $\mathrm{Cu}(\mathrm{K} \boldsymbol{\alpha})$ radiation $(\lambda$ $=1.5406 \AA$ ).

In-situ $x$-ray diffraction: The in-situ x-ray diffraction measurements were carried out in the in the q-range between 0.2 and $1.6 \AA^{-1}$ corresponding to $2 \theta$ between $3^{\circ}$ and $15^{\circ}$ with a $0.03^{\circ}$ step and speed of 4 degrees per minute under the operation conditions of $40 \mathrm{KV}$ and $35 \mathrm{~mA}$. For this experiment, the samples were prepared by spin coating the precursor solution onto glass at 2500 r.p.m for $1 \mathrm{~s}$. The spin-coating conditions were optimized to obtain the most uniform substrate. The fabricated wet film was immediately transferred to the x-ray diffraction instrument. The film growth was monitored over time for up to 120 minutes. The diffraction measurements were continuously recorded until the film is dried and the diffraction peaks were saturated. The peaks in the obtained diffraction patterns were assigned Miller indices based on the simulated diffraction pattern from the structural data available from our previous work. ${ }^{25,26}$

\subsection{Grazing incidence wide angle $x$-ray scattering (GIWAXS)}

Experimental methods: All GIWAXS diffraction spectra used in this paper were high-resolution synchrotron patterns measured at two beamlines: 8-ID-E at the Advanced Photon Source (APS) and 11-BM at the National Synchrotron Light Source-II (NSLS II). At beamline 8-ID-E, samples were placed on a Linkam grazing incidence $\mathrm{x}$-ray-scattering (GIXS) stage (temperature controlled at $\left.25^{\circ} \mathrm{C}\right)$ inside a vacuum chamber $\left(10^{-4}\right.$ torr $)$ with the sample $228 \mathrm{~mm}$ away from a Pilatus $1 \mathrm{M}$ (Dectris) area detector. The photon energy was $10.91 \mathrm{keV}$, and the beam size was $200 \mu \mathrm{m}$ x $20 \mu \mathrm{m}$ $(\mathrm{H} \mathrm{x} \mathrm{V})$. At beamline 11-BM, samples were placed on a robotic stage inside a vacuum chamber $\left(10^{-2}\right.$ torr) with the sample $267 \mathrm{~mm}$ away from a Pilatus $800 \mathrm{~K}$ (Dectris) area detector. The photon energy was $13.5 \mathrm{keV}$, and the beam size was $200 \mu \mathrm{m}$ x $50 \mu \mathrm{m}(\mathrm{H} \mathrm{x} \mathrm{V})$.

GIWAXS analysis: The GIWAXS data were processed using the GIXSGUI package (version 1.7.3) running on Matlab 2018b (Mathworks). All patterns were corrected with conditions such as detector sensitivity, X-ray polarization, and geometrical solid-angle parameters. Sector-cut integration was taken from the GIWAXS map and used for general analysis. The diffraction patterns were analyzed using the structural characteristics of 2D perovskite crystals reported in our previous work. ${ }^{33}$ The software SingleCrystal by CrystalMaker was used to simulate the x-ray diffraction of 2D perovskite crystals and assign Miller indices to diffraction peaks in the GIWAXS patterns (see example in Fig. 1b-d). The details of the procedure is described in Supplementary Fig. 2 and 3 and involves simulating the diffraction of crystal with different orientation [uvw] with respect to the incident $\mathrm{x}$-ray beam and ponder the weight of each pattern in the experimental data. 
Crystallinity analysis: The Debye Scherrer analysis was used to derive the average grain size of the parent-crystals and thin films. ${ }^{59}$ The Bragg (111) and (011) diffraction peaks of RuddlesdenPopper and Dion-Jacobson phase perovskites were used for the analysis. The shape factor of 0.9 ( $\mathrm{K}$ value in the Scherrer equation) was used. The Scherrer formula is:

$$
D_{h k l}=\frac{K \lambda}{\beta_{h k l} \cos \left(\theta_{h k l}\right)}
$$

where, $\theta_{h k l}$ is the Bragg scattering angle and $\beta_{h k l}$ is the full-width-at-half-max (FWHM). Since this is a grazing scattering geometry with area detectors, we have corrected the FWHM for the beam divergence $\left(\beta_{\text {div }}\right)$ and the energy bandwidth $\left(\beta_{B W}\right){ }^{60}$

$$
\begin{gathered}
\beta_{\text {hkl }}=\sqrt{\beta_{\text {experiment }}^{2}-\beta_{\text {res }}^{2}} \\
\beta_{\text {res }}=\sqrt{\beta_{\text {div }}^{2}+\beta_{B w}^{2}}
\end{gathered}
$$

\subsection{Optical Absorbance and Photoluminescence measurements}

Single crystal absorbance: The absorbance of the parent-crystals was conducted on individual thin (less than $100 \mathrm{~nm}$ thick) single crystals mechanically exfoliated on glass substrates inside a Arglovebox. The samples were kept under vacuum $\left(10^{-5}\right.$ Torr $)$ and at room temperature during the measurements. Optical absorbance measurements were performed by focusing with a microscope a white-light LED (Thorlabs SOLIS-3C) and measuring the transmitted light spectrum using a spectrograph (Andor Kymera) and a CCD (Andor iDus) camera.

Thin film absorbance: Film absorbance measurements were performed by illuminating the samples with monochromatic light modulated at $2 \mathrm{kHz}$ coming from a quartz-tungsten-halogen light source fed into a monochromator (SpectraPro HRS 300, Princeton instruments). Synchronous detection of the transmitted light was achieved with a silicon photodiode hooked to a SR865 lock-in amplifier. During all measurements, the samples were kept under vacuum $\left(\sim 10^{-4}\right.$ torr $)$ and at room temperature.

In-situ absorbance measurements: The in-situ absorbance of the thin film measured during film formation were performed using a white-light LED (Thorlabs SOLIS-3C). The transmitted light spectrum was measured using a spectrograph (Andor Kymera) and a CCD (Andor iDus) camera. After spin coating the precursor solution onto glass substrate in a Ar-glovebox, we directly transferred our sample to our absorbance apparatus and monitored the transmitted light during thin film formation. The measurement was taken every 1 minute for 2 hours.

\subsection{Dynamic Light Scattering measurements}

Experimental methods: Precursor solutions (phase-selective and classic) were prepared at a concentration of 0.4 M in DMF (DMF, Protein Technologies Inc. AZ 85714) and subsequently loaded into cylindrical glass cuvettes (3mm diameter, LS Instruments AG, Fribourg). Dynamic light scattering correlation curves were immediately collected at five directions with respect to the incident laser beam corresponding to the angles $30^{\circ}, 60^{\circ}, 90^{\circ}, 120^{\circ}, 150^{\circ}$ (or at a single angle over $\sim 40 \mathrm{hrs}$ ), and at ambient temperature $21^{\circ} \mathrm{C}$ using a fully automated 3D LS Spectrometer (LASER: $660 \mathrm{~nm}, 65 \mathrm{~mW}$, LS Instruments AG, Fribourg). 
Extraction of the size of the particles in solution: Detailed multi-angle DLS analysis of precursor solutions is provided below. For each solution, the angular dependence of the autocorrelation function was measured. Curves were fitted using a single- or bi-exponential decay model and the resulting correlation fitting residuals were plotted, followed by a linear regression of $\Gamma$ vs $q^{2}$ between $30^{\circ}$ and $150^{\circ}$.

The scattered light correlation function, $g^{(2)}{ }^{61}$ compares and average the intensity of received signal at time $\mathrm{t}$ with that of signal at later times later $\mathrm{t}+\tau$,

$$
g^{(2)}(q, \tau)=\frac{<I(t) I(t+\tau)>}{<I(t)^{2}>}
$$

The intensity correlation function is then related to the field correlation function using the Siegert relationship ${ }^{62}$

$$
g^{(2)}(q, \tau)-1=\beta\left|g^{(1)}(q, \tau)\right|^{2},
$$

where $\beta$ is a constant proportional to the signal-to-noise ratio.

For a system undergoing Brownian motion the electric field correlation function is shown to decay exponentially as ${ }^{63}$

$$
g^{(1)}(q, \tau)=\exp (-\Gamma \tau)
$$

When several groups of particles of different sizes (labelled $i$ ) are present in solution, the DLS data can be fitted using:

$$
g^{(2)}(q, \tau)-1=\sum_{i} \alpha_{i}\left|g_{i}^{(1)}(q, \tau)\right|^{2}=\sum_{i} \alpha_{i}\left|\exp \left(-\Gamma_{i} \tau\right)\right|^{2} .
$$

Then, for the group of particles $i$ the value $\Gamma_{i}$ is related to the translational diffusion coefficient $D_{T, i}$ and wave number $q$ through $^{64}$

with,

$$
\Gamma_{i}=D_{T, i} q^{2}
$$

$$
q=\frac{4 \pi \eta}{\lambda} \sin \left(\frac{\theta}{2}\right)
$$

$\eta$ being the refractive index of the solvent, $\lambda$ the wavelength of the laser, and $\theta$ the angle between the incident laser beam and the scattered one. Finally, the diffusion coefficient is related to the hydrodynamic radius $R_{\mathrm{H}}$ of particles in a Brownian motion by the Stokes-Einstein equation ${ }^{65}$

$$
D_{T, i}=\frac{k_{\mathrm{B}} T}{6 \pi \mu R_{\mathrm{H}, i}},
$$

with $k_{\mathrm{B}}$ being the Boltzmann constant, $T$ the temperature, $\mu$ the dynamic viscosity, and $R_{H, i}$ the median hydrodynamic radius of the group $i$ of particles.

\subsection{Bright-field imaging of the thin film formation}

Bright-field imaging was performed using an Olympus IX83 fully automated inverted microscope (Olympus Corporation, Tokyo) and an Electron Multiplying Charge-Coupled Device (EMCCD) camera (ANDOR iXon Ultra, Andor Technology, Belfast). An oil-immersion 100X objective (Olympus UAPO N 100X, Olympus Corporation, Tokyo) was used to perform in-situ imaging of the thin film formation by taking snapshot images of the sample every second ( $1 \mathrm{fps})$. For this 
experiment we used a precursor solution with concentration of $0.4 \mathrm{M}$. Few microliters of the solution was dropped onto a glass slide and the imaging of the film formation was started directly afterwards. A 20X objective (Olympus UPLFLN 20X, Olympus Corporation, Tokyo) was used to image the thin film formation. The images were processed with ImageJ (ImageJ 1.8.0_112, NIH, MD).

\subsection{Solar cell devices characterization}

Solar cell performances: The performances of the fabricated solar cells were characterized by measuring the current-voltage (J-V) curves of each device illuminated by an ABB solar simulator from Newport (model 94011). The arc simulator modeled AM 1.5G irradiance of $100 \mathrm{~mW} / \mathrm{cm}^{2}$ and was calibrated using a NIST-certified Si solar cell (Newport 91150V, ISO 17025). The current was measured with a Keithley 2401 instrument at different voltage scan rate.

External quantum efficiency: The external quantum efficiency of the solar cell devices was collected by first illuminating each device with monochromatic light modulated at $2 \mathrm{kHz}$ coming from a quartz-tungsten-halogen light source fed into a monochromator (SpectraPro HRS 300, Princeton instruments). The photocurrent response of the solar cells was measured by a SR865 lock-in amplifier. The light source spectrum response was calibrated using a calibrated silicon diode (FDS1010, Thorlab).

Stability tests: For stability test measurements, the perovskite devices were encapsulated with a UV- curable epoxy (Poland Inc.) and a glass coverslip as a barrier layer in an argon-filled glove box. The devices were blown with the argon gun in order to remove any contaminants or dust particles just before encapsulation. All the devices were tested under open-circuit (OC) conditions, under full-spectrum simulated AM 1.5G (100 $\mathrm{mA} \mathrm{cm}^{-2}$ irradiance) in air using an ABB solar simulator (94011A, Newport) - ISOS-L protocol. The relative humidity was measured to be constant at $60 \pm 5 \% \mathrm{RH}$.

\section{Additional references}

59. Patterson, A. L. The Scherrer Formula for X-Ray Particle Size Determination. Phys. Rev. 56, 978-982 (1939).

60. Smilgies, D.-M. Scherrer grain-size analysis adapted to grazing-incidence scattering with area detectors. J. Appl. Crystallogr. 42, 1030-1034 (2009).

61. Ross-Murphy, S. B. Dynamic Light Scattering. B. J. Berne and R. Pecora, John Wiley, New York, 1976, pp. 376. Price £16.50. Br. Polym. J. 9, 177-177 (1977).

62. Siegert, A. J. F. On the fluctuations in signals returned by many independently moving scatterers. (Radiation Laboratory, Massachusetts Institute of Technology, 1943).

63. Carpenter, D. K. Dynamic Light Scattering with Applications to Chemistry, Biology, and Physics (Berne, Bruce J.; Pecora, Robert). J. Chem. Educ. 54, A430 (1977).

64. Stetefeld, J., McKenna, S. A. \& Patel, T. R. Dynamic light scattering: a practical guide and applications in biomedical sciences. Biophys. Rev. 8, 409-427 (2016).

65. Einstein, A. Über die von der molekularkinetischen Theorie der Wärme geforderte Bewegung von in ruhenden Flüssigkeiten suspendierten Teilchen. Ann. Phys. 322, 549-560 (1905). 


\section{Supplementary Figures and Related discussion}
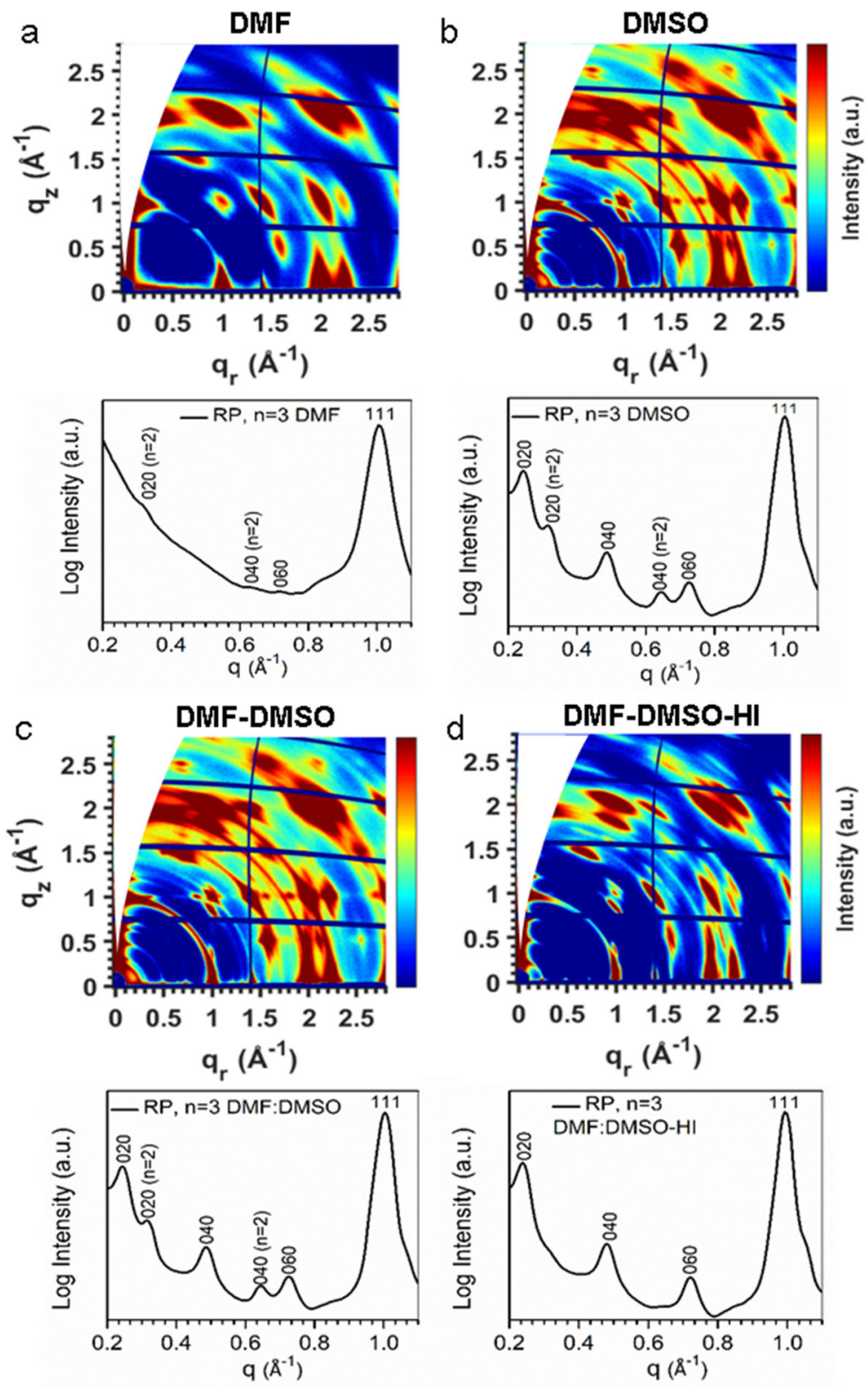

Supplementary Fig. 1 | Structural characterization of the Ruddlesden-Popper $\mathrm{BA}_{2} \mathbf{M A}_{2} \mathrm{~Pb}_{3} \mathbf{I}_{10}$ perovskite thin films prepared with the phase-selective synthesis method using different types of solvent. a to d present the GIWAXS results for the solvents DMF, DMSO, DMF-DMSO, DMF-DMSO-HI, respectively. Structure of the film synthesized with the solvent DMF-MACl is presented in Fig. 1c. In each panel, the top image graph is the GIWAXS pattern and the bottom plot is the diffraction derived from integration of the GIWAXS data over the full azimuthal angle. The Miller indices for the desired $n=3$ phase are indicated as hkl, while phase impurities (i.e. phases with different 2D perovskite layer thicknesses or $n$-values) are indicated as $\mathrm{hkl}_{(\mathrm{n})}$. 

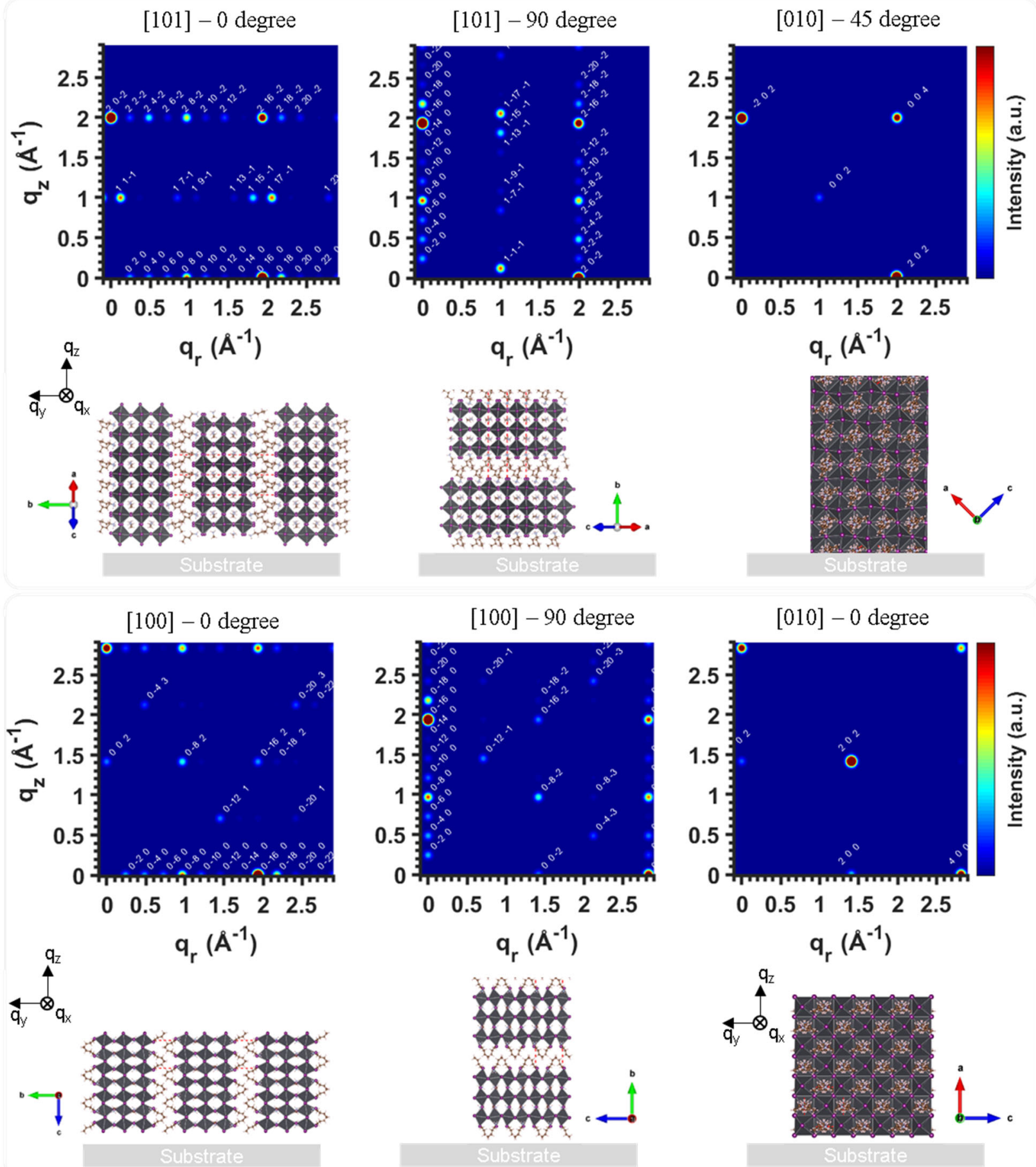

Supplementary Fig. 2 | Simulation method of the GIWAXS pattern for a RuddlesdenPopper- Simulated $\mathrm{BA}_{2} \mathrm{MA}_{2} \mathrm{~Pb}_{3} \mathrm{I}_{10}$ single crystal diffraction pattern with different orientation with respect to the X-ray incident beam. The intensity maps show the patterns for different [uvw] crystal direction and orientation (angle in degrees from the normal to the substrate). The crystal structure below each pattern sketch the corresponding crystal direction/orientation with respect to the substrate and $\mathrm{x}$-ray beam that is set along the $\mathrm{q}_{\mathrm{x}}$ direction. $\left(\mathrm{q}_{\mathrm{r}}, \mathrm{q}_{\mathrm{z}}\right)$ are the cylindrical coordinates for the Cartesian base $\left(\mathrm{q}_{\mathrm{x}}, \mathrm{q}_{\mathrm{y}}, \mathrm{q}_{\mathrm{z}}\right)$. 


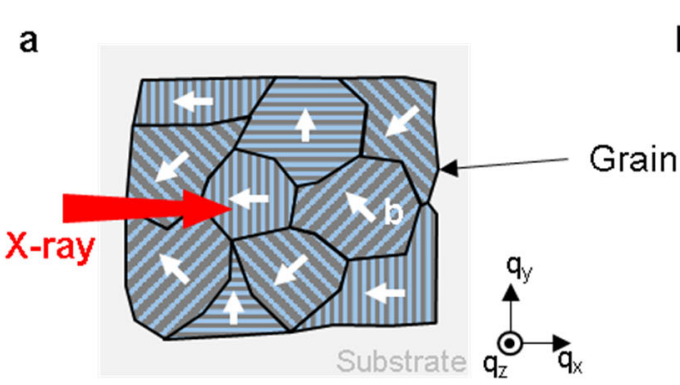

Top view of thin film

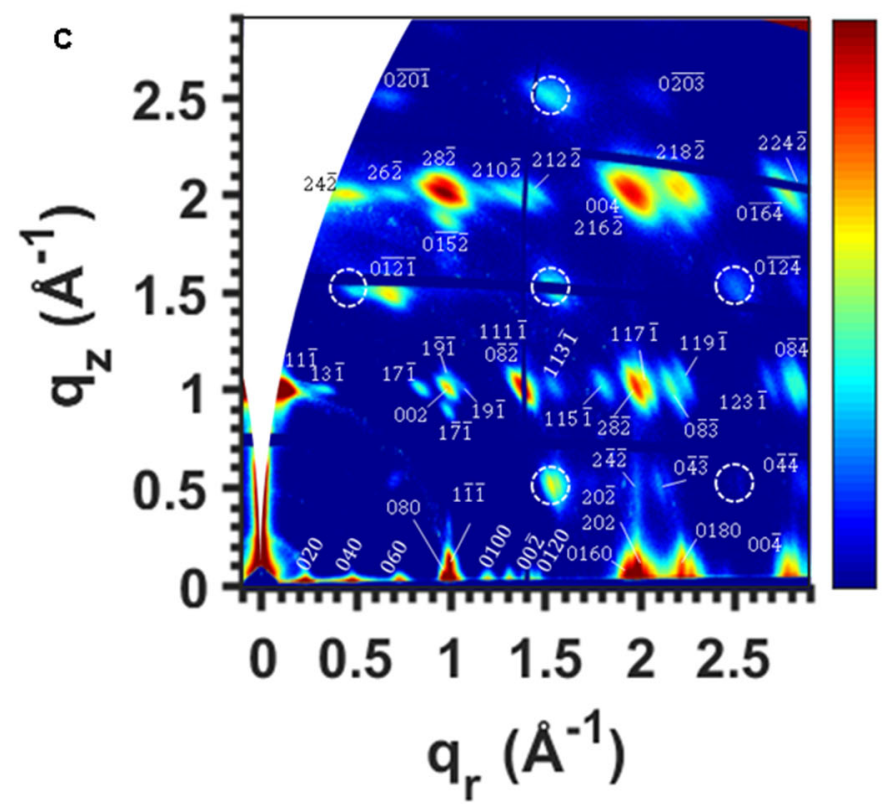

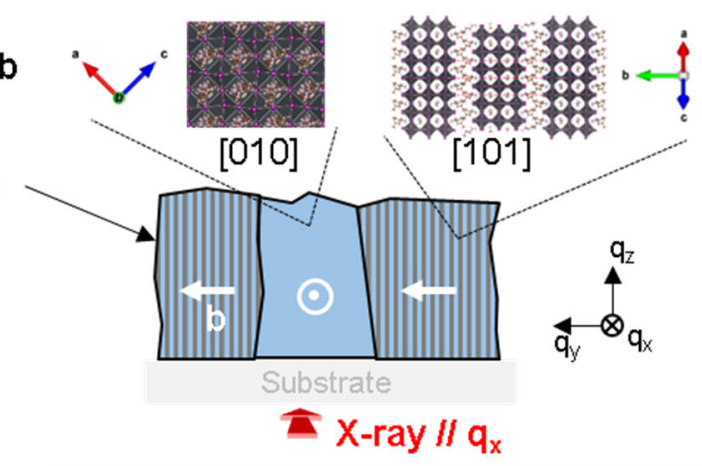

Side view of film from $x$-ray beam direction

Supplementary Fig. 3 | Configuration of 2D perovskite thin films in the GIWAXS apparatus and detailed assignment of the diffraction planes for the GIWAXS of the $\mathrm{BA}_{2} \mathrm{MA}_{2} \mathrm{~Pb}_{3} \mathrm{I}_{10}$ thin films. We set the direction of the $\mathrm{x}$-ray beam along $\mathrm{q}_{\mathrm{x}}$. The grains composing the films are formed of $2 \mathrm{D}$ perovskite crystal stacked along the $\mathbf{b}$-direction. a, In the phase-selective films prepared with DMF-MACl solvent, most of the perovskite layers are oriented normal to the substrate as sketched here, however the stacking axis of each grain is randomly oriented in the plane of the substrate. $\mathbf{b}$, Given the fixed direction of the x-ray beam, most of the diffraction intensity measured in our GIWAXS experiments stem from grains with stacking direction parallel to the beam (corresponding to the [010] crystal direction) and perpendicular to the beam (corresponding to the [101], [100], or [001] crystal directions), see also thin film crystal structure in Fig. 1e. c, GIWAXS pattern of the $\mathrm{BA}_{2} \mathrm{MA}_{2} \mathrm{~Pb}_{3} \mathrm{I}_{10}$ thin films with all the Miller indices derived from the simulated data in the Supplementary Fig. 2. d, Corresponding diffraction pattern. The dashed circles indicate the peaks that cannot be reproduced as discussed in the main text. 


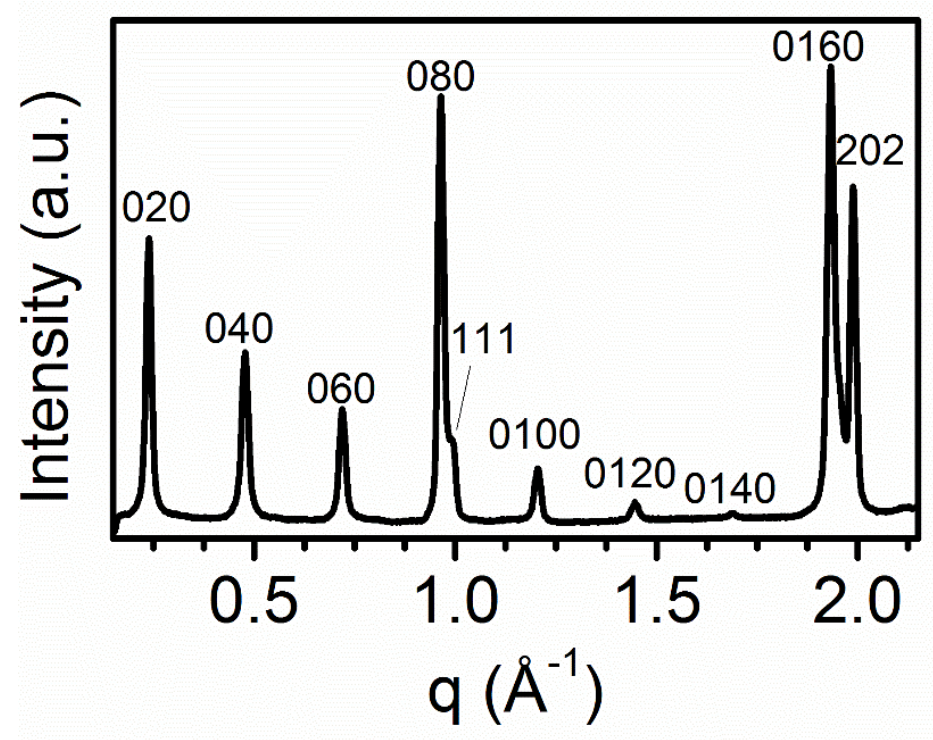

Supplementary Fig. 4 X-ray diffraction pattern of the crushed powder scrapped from the oriented $\mathrm{BA}_{2} \mathrm{MA}_{2} \mathrm{~Pb}_{3} \mathrm{I}_{10}$ films indicating phase purity.

a

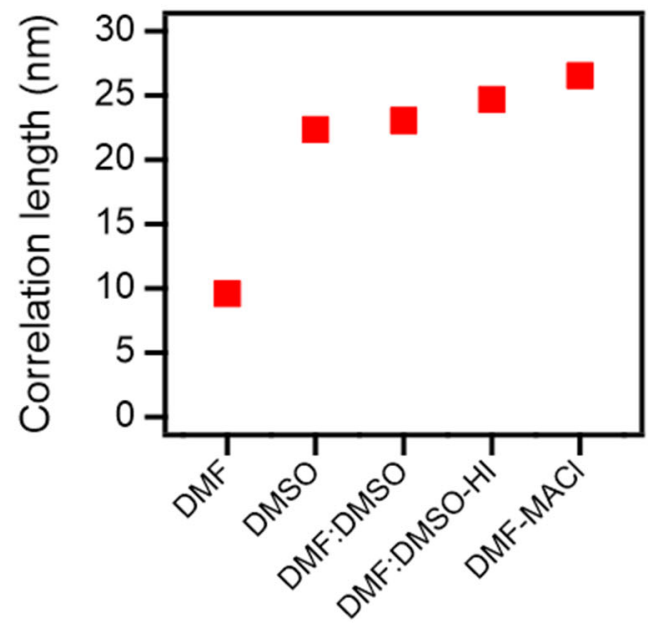

b

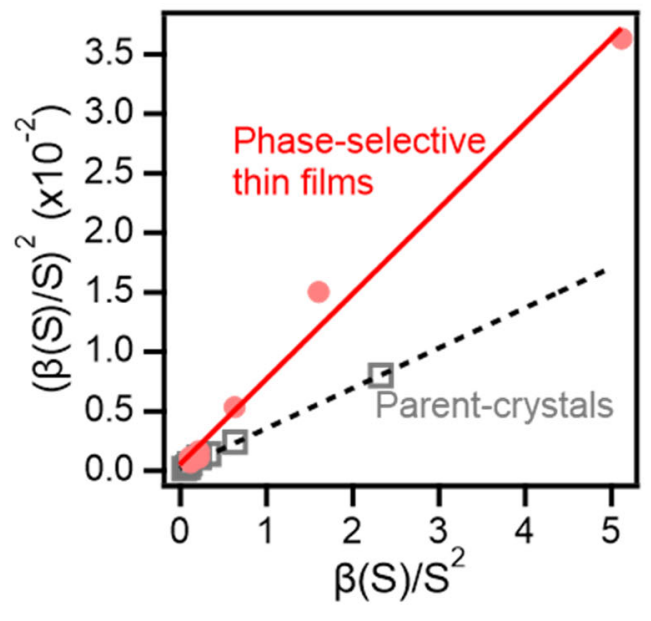

Supplementary Fig. 5 | Grain size analysis of the $\mathbf{B A}_{2} \mathbf{M A}_{2} \mathbf{P b}_{3} \mathbf{I}_{10}$ thin films. a, Debye Scherrer analysis of the average gran size in the films prepared with different solvents. The best grain size is obtained for the DMF-MACL film. b, Average grain size of the phase selective film with DMFMACL and of the parent crystal. 

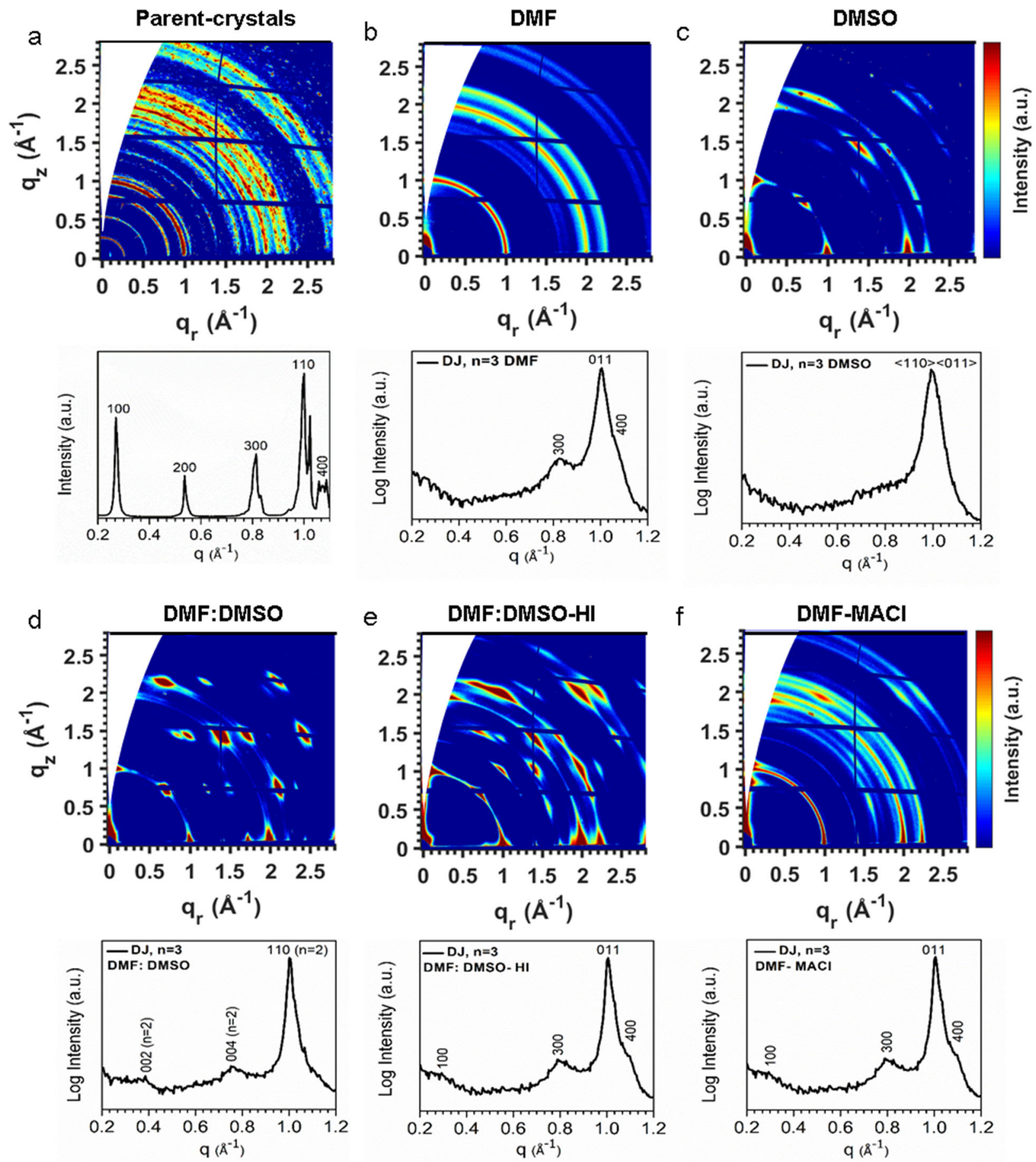

Supplementary Fig. 6 | Structural characterization of the Dion-Jacobson 4AMP-MA2 $\mathrm{Pb}_{3} \mathrm{I}_{10}$ perovskite parent-crystals and thin films prepared with the phase-selective synthesis method using different types of solvent. a, GIWAXS pattern for the parent-crystal. $\mathbf{b}$, to f, present the GIWAXS results for the solvents DMF, DMSO, DMF-DMSO, DMF-DMSO-HI, and DMF$\mathrm{MACl}$, respectively. In each panel, the top image graph is the GIWAXS pattern and the bottom plot is the diffraction derived from integration of the GIWAXS data over the full azimuthal angle. 
The Miller indices for the desired $n=3$ phase are indicated as hkl, while phase impurities (i.e. phases with different $2 \mathrm{D}$ perovskite layer thicknesses or $n$-values) are indicated as $\mathrm{hkl}_{(\mathrm{n})}$. The best combination of phase purity, crystallinity and preferential orientation in the $4 \mathrm{AMP}-\mathrm{MA}_{2} \mathrm{~Pb}_{3} \mathrm{I}_{10}$ are obtained with the DMF-DMSO-HI solvent condition.

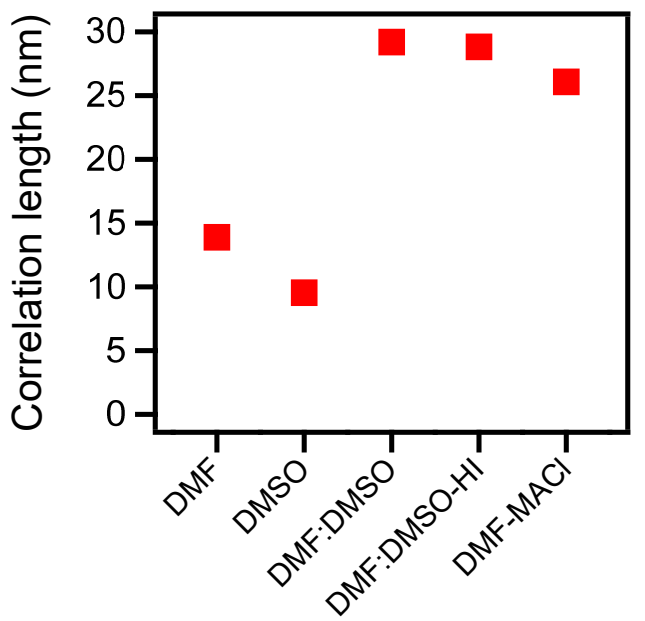

Supplementary Fig. 7 Grain size analysis of the Dion-Jacobson 4AMP-MA $\mathbf{M A}_{2} \mathbf{P b}_{3} \mathbf{I}_{10}$ perovskite thin films. Average grain size corresponding to the films prepared with different solvent, as derived from the Debye Scherrer equation. 

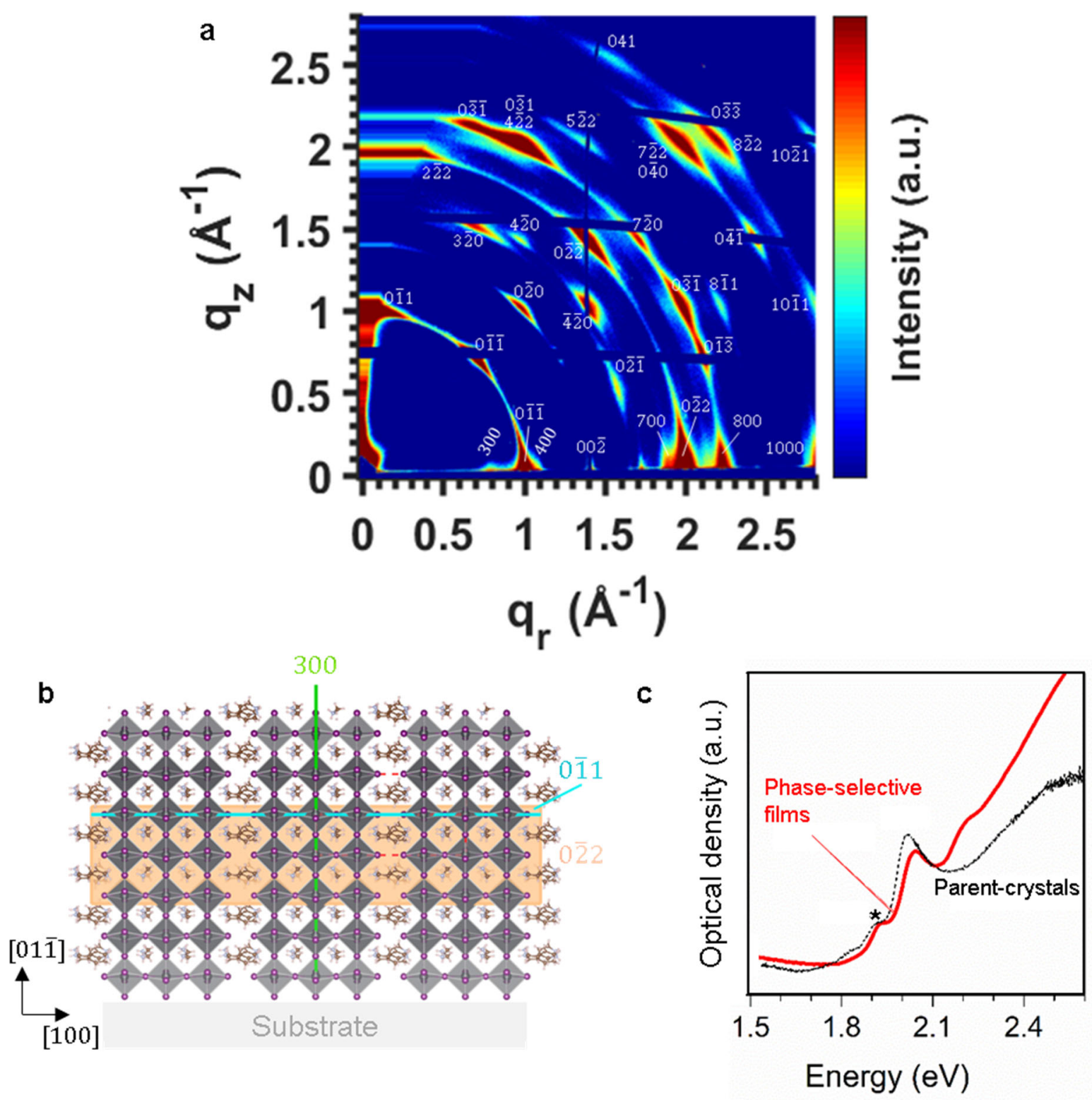

Supplementary Fig. 8 | Detailed structural characterization of the optimized 4AMP$\mathbf{M A}_{2} \mathbf{P b}_{3} \mathbf{I}_{10}$ films prepared with the DMF:DMSO-HI solvent. a, Grazing incidence wide-angle $\mathrm{x}$-ray scattering patterns of the thin films synthetized with the phase-selective method. Labels indicate the Miller indices indexed from the 2D perovskite single crystal structure with principally vertically oriented perovskite layers as sketched in $\mathbf{b}$. $\mathbf{c}$, Optical absorbance spectra of the films as compared to the parent-crystals. The star indicate the presence of a small amount of $n=4$ impurity phase, which was already present in the parent-crystals. 


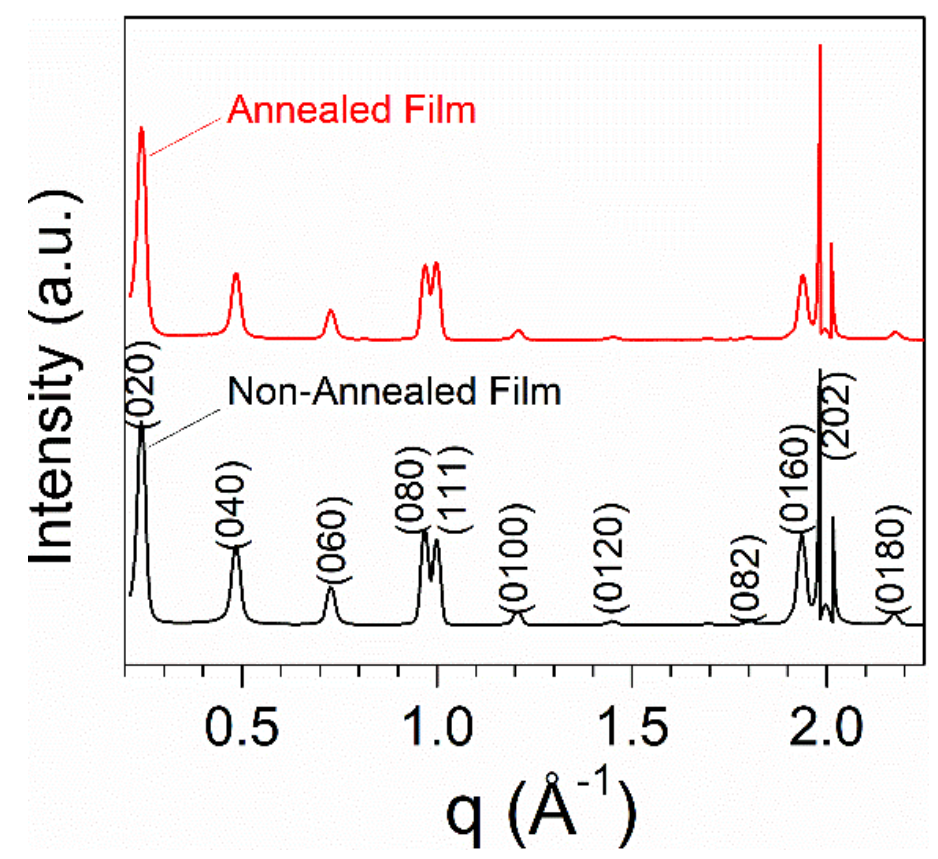

Supplementary Fig. 9 | Comparison of the x-ray diffraction of the $\mathrm{BA}_{2} \mathrm{MA}_{2} \mathrm{~Pb}_{3} \mathrm{I}_{10}$ film used for the in-situ experiments (non-annealed film) to the thin films obtained after annealing.

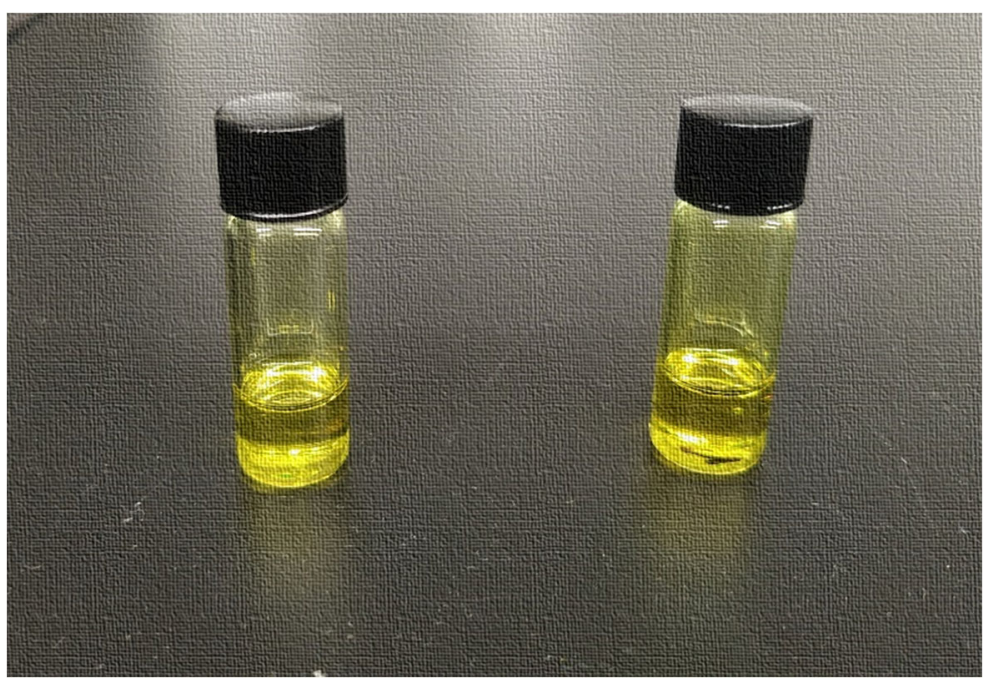

Supplementary Fig. 10 | Pictures of the typical precursor solutions prepared in the classic synthesis method (left) and the phase-selective synthesis method (right). No differences in color or clarity was observed from inspection with our eyes. 

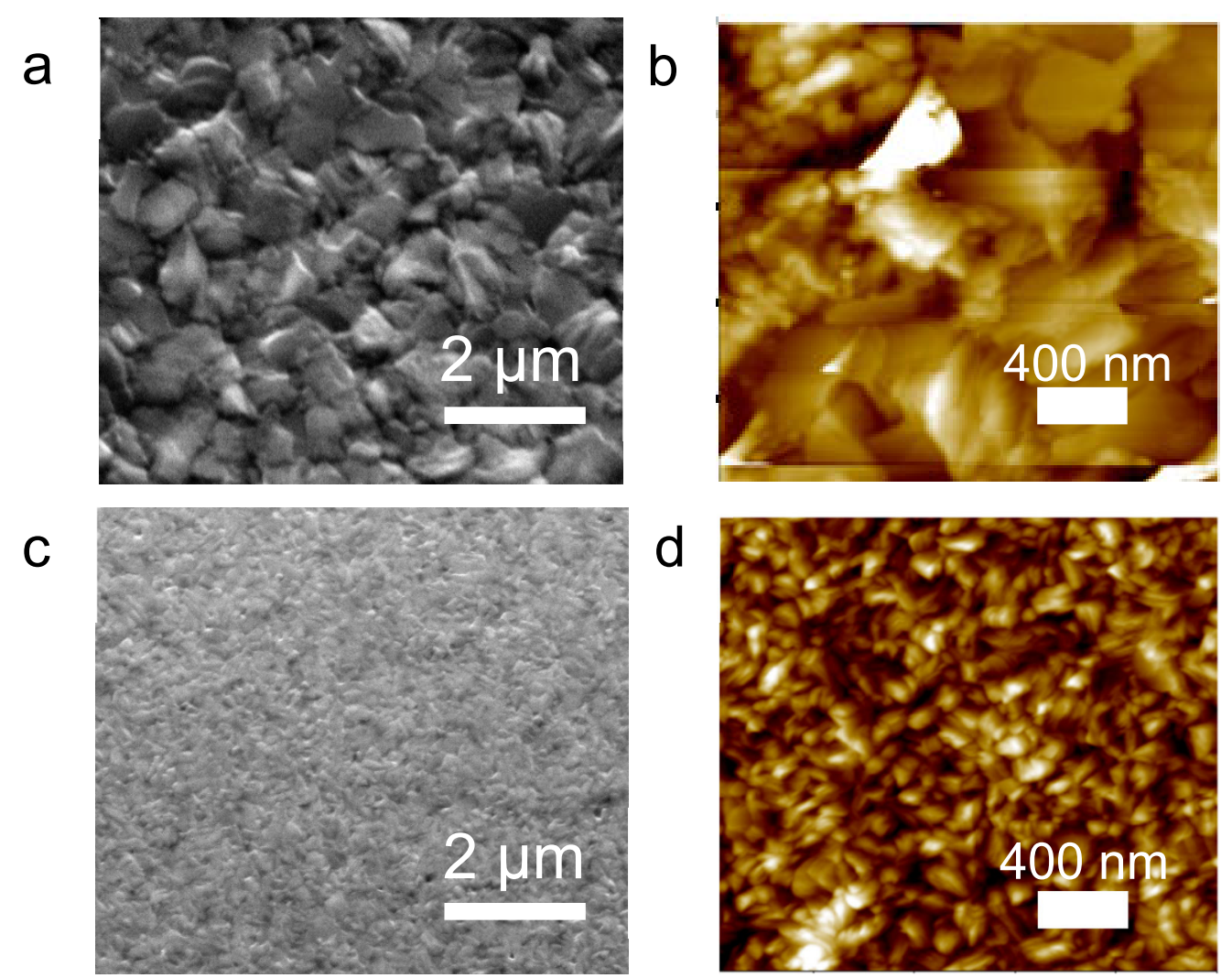

Supplementary Fig. 11 Surface morphology of the $\mathbf{B A}_{2} \mathbf{M A}_{2} \mathrm{~Pb}_{3} \mathbf{I}_{10}$ thin films synthetized via (a, b) the phase-selective method and (c, d) the classic method. a and c were obtained from scanning electron microscopy. $\mathrm{b}$ and $\mathrm{d}$ are images from atomic force microscopy. 

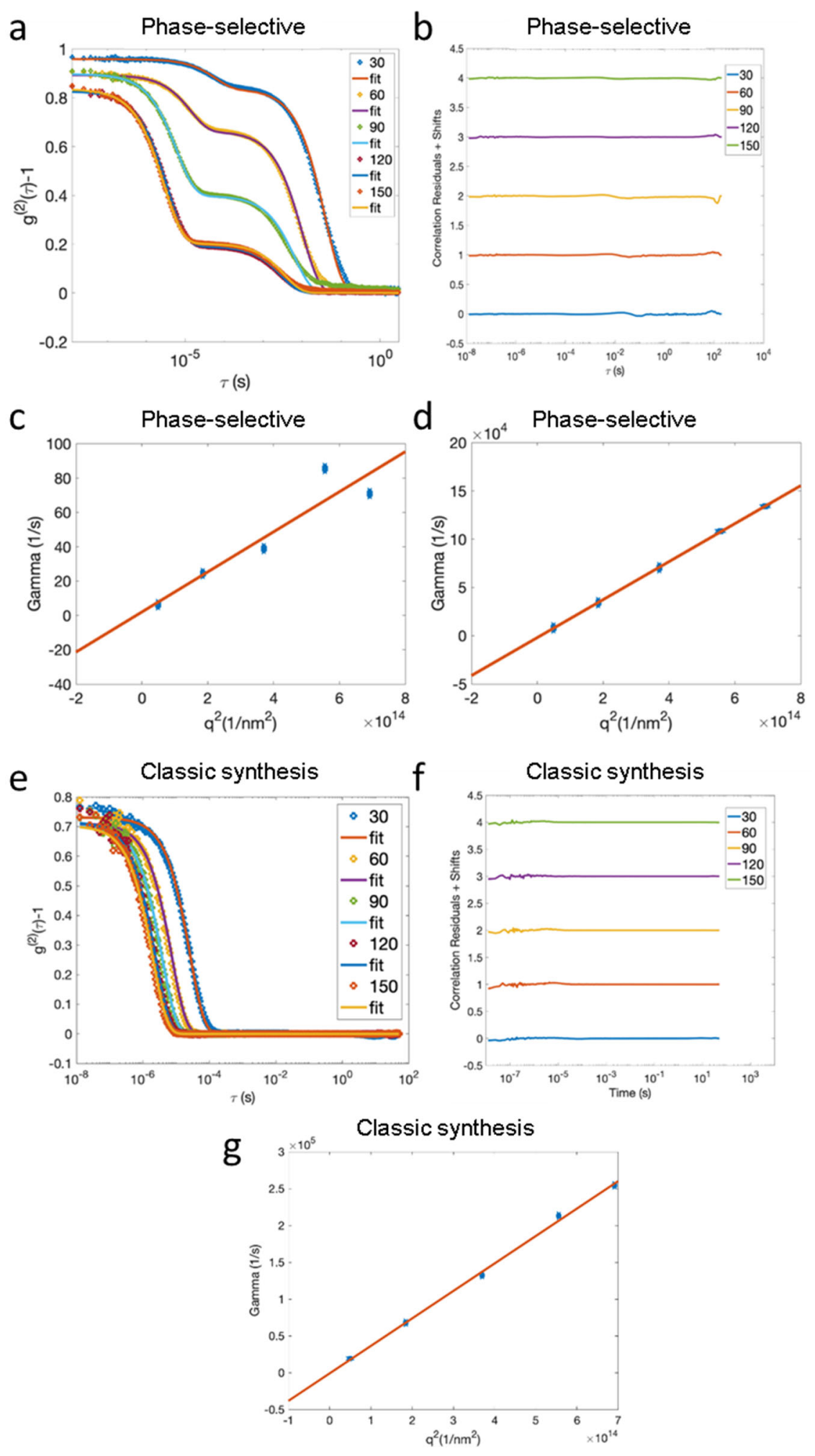

Supplementary Fig. 12 | Size determination by dynamic light scattering of particles present in the precursor solutions for the phase-selective and classic synthesis processes. a, e, Correlation function $\left(\mathrm{g}^{(2)}\right)$ versus lag time $(\tau)$ measured at several scattering angles. The lines 
correspond to the fit to the data. $\mathbf{b}, \mathbf{f}$, Residual of the fit functions. $\mathbf{c}, \mathbf{d}$, and $\mathbf{g}$, Corresponding linear regression of the Gamma values $(\Gamma)$ derived from the fit to the data. In the case of the phaseselective method, C and D correspond to the Gamma value of the small and large particles, respectively, as derived from the bi-exponential fit to the data in A. In the case of the classic synthesis method, the precursor solution contains only one type of particles of small size, thus the data in $\mathrm{E}$ were fitted with a single exponential function.

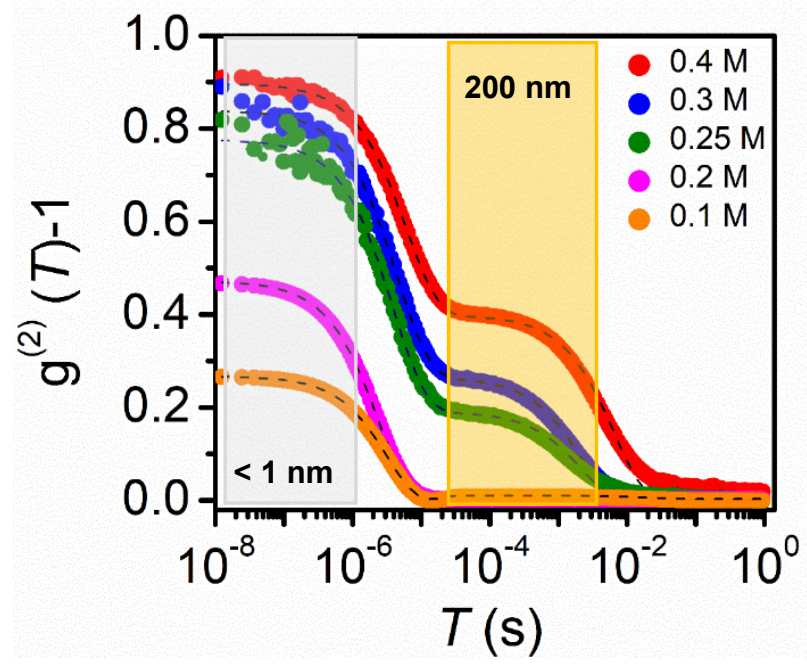

Supplementary Fig. 13 | Correlation function versus delay time derived from dynamic light scattering performed for different concentration of the perovskite solution for the phase-selective method. Dashed lines are fit to the data. Gray and orange regions indicate small and large particle size in solution, respectively.

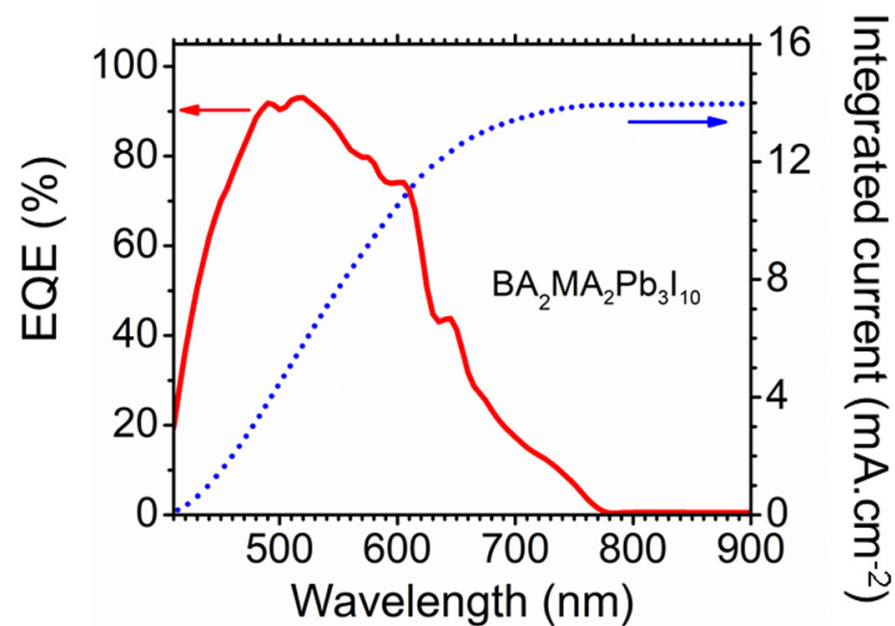

Supplementary Fig. 14 | External quantum efficiency of the Ruddlesden-popper n=3 perovskite solar cells. 


\section{Figures}
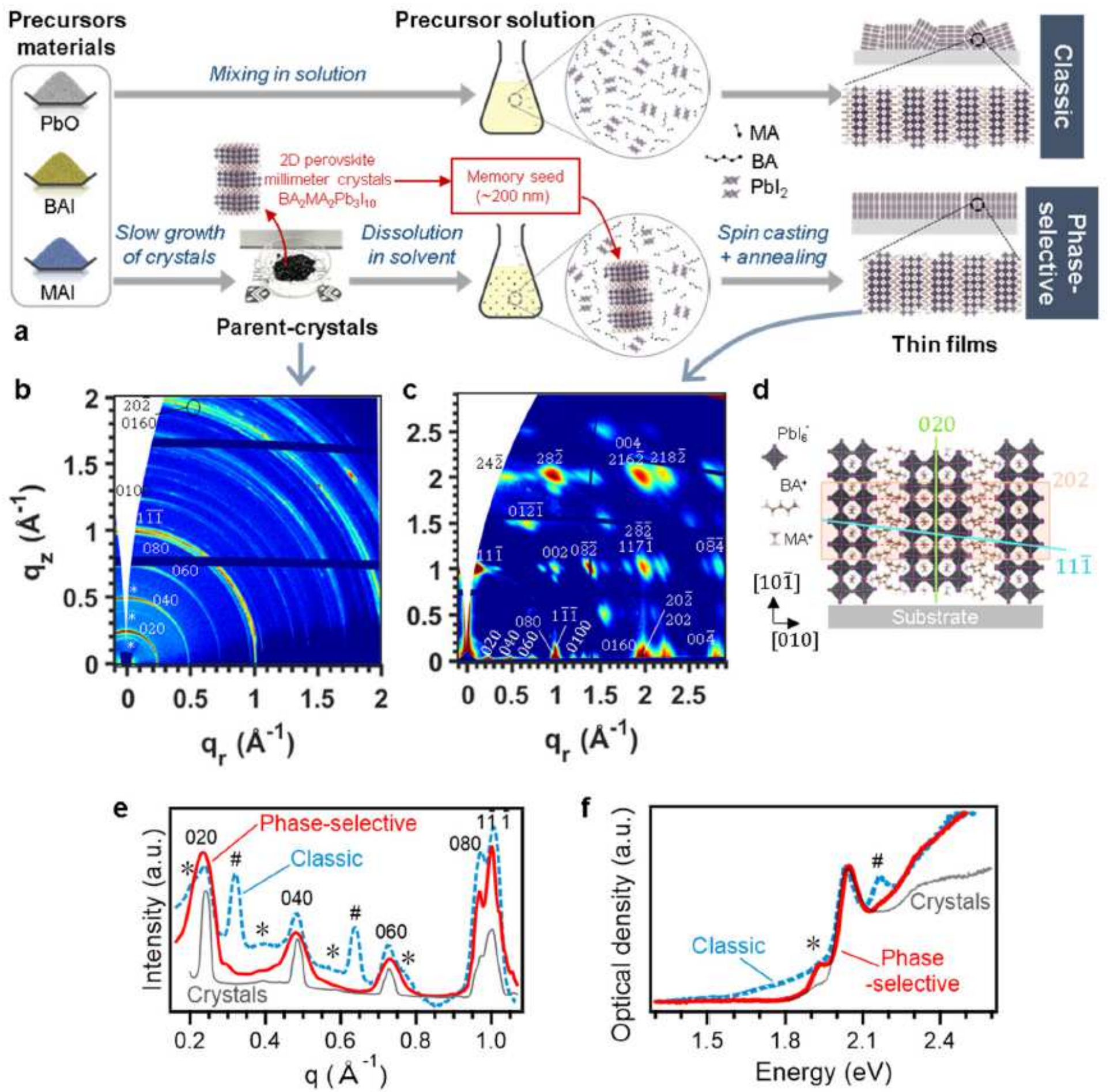

\section{Figure 1}

Phase-selective synthesis method to achieve high phase-purity in 2D perovskite thin-films. a, Sketch of the phase-selective synthesis method from this work and the classic synthesis method already reported in the literature. Example of the Ruddlesden-Popper BA2MA2Pb3I10 2D perovskites here. Grazing incidence wide-angle $x$-ray scattering patterns of $b$, the parent-crystal powders and $c$, the thin films 
synthetized with the phase-selective method. Labels indicate the Miller indices of the principal diffraction peaks derived from the simulated diffraction pattern of the thin films with convention and plane directions indicated in d (see details in Supplementary Fig. 2 and 3). e, Angular-integrated diffraction patterns of the thin films prepared with the phase-selective and classic synthesis methods, and comparison to the parentcrystals. $f$, Corresponding optical absorbance spectra. Here, the star and hash symbols identify the $n=4$ and $n=2$ impurity phases.
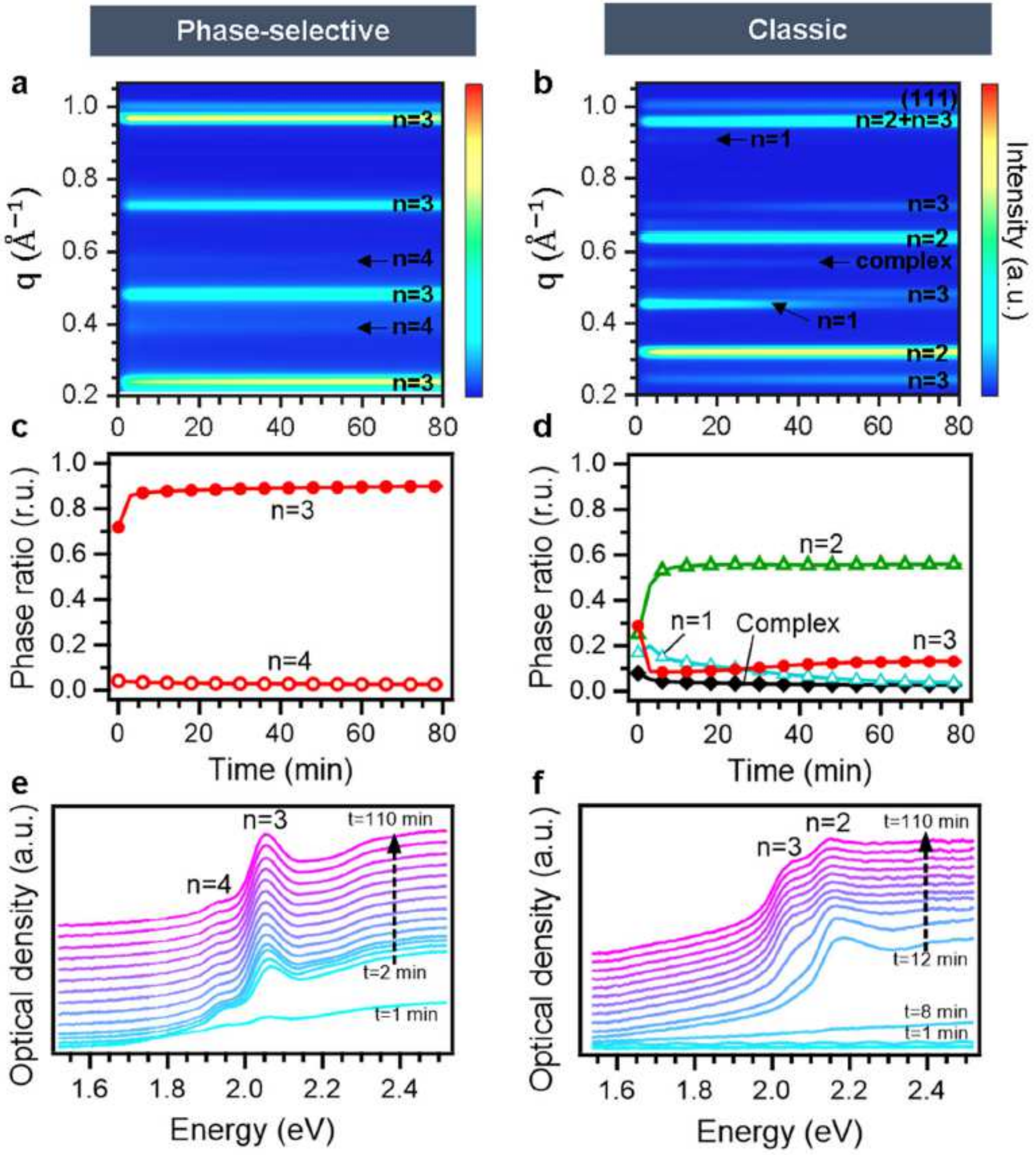

Figure 2 
In-situ structural and absorption characterization of the film formation in Ruddlesden-Popper $n=3$ perovskite. a, b, Evolution of the x-ray diffraction during film formation and comparison between our phase-selective synthesis method and the classic one. All peaks were identified to a 2D perovskite phase of defined n-value (or perovskite layer thickness), except for one peak assigned to the presence of an intermediate complex in solution. $c, d$, Corresponding evolution of the ratio of each phase relative to the fully integrated diffraction. e, f, Evolution of the optical absorbance spectra of the films during synthesis. Each peak in the spectra correspond to the ground exciton transition of a given perovskite layer thickness.
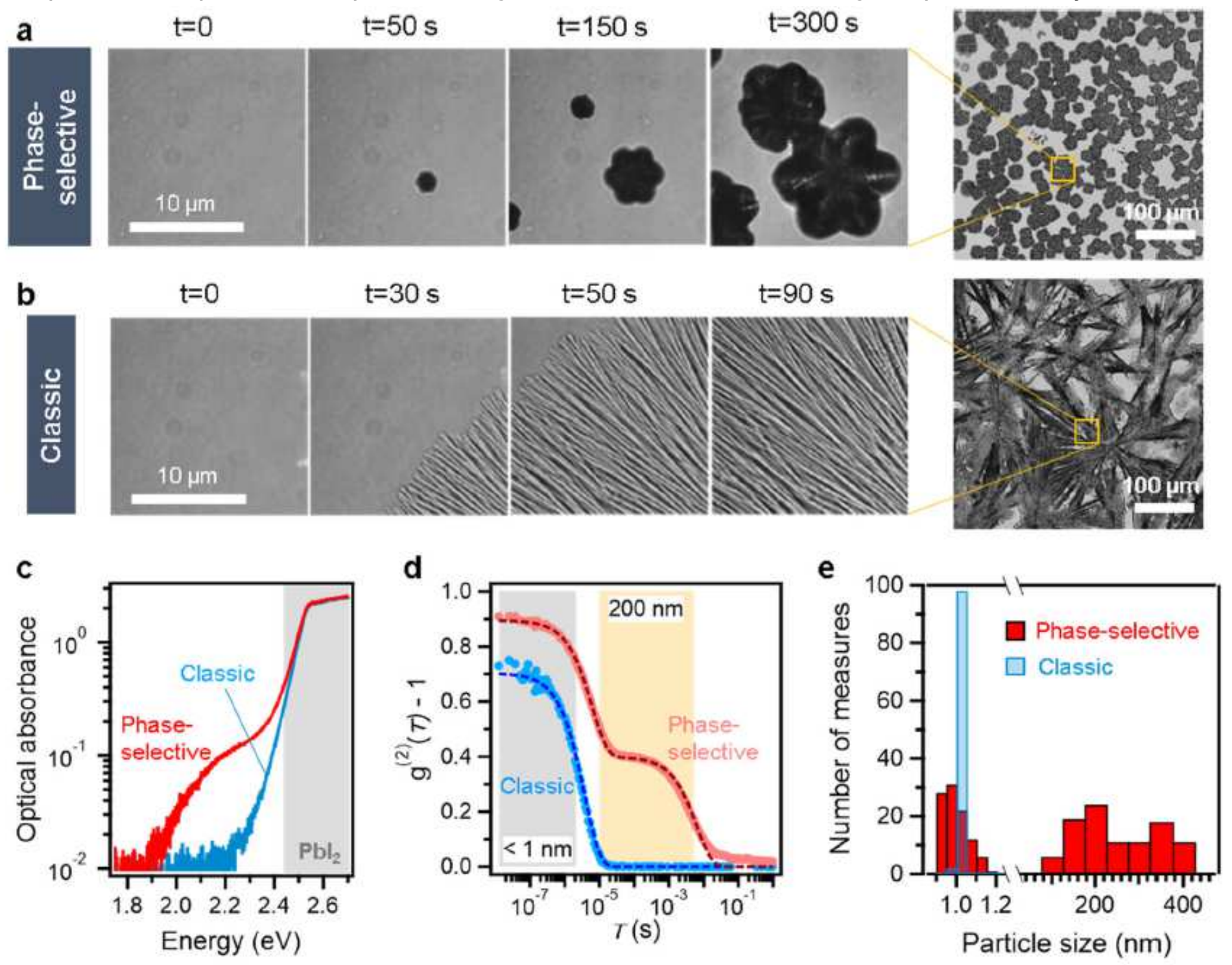

f

Phase-selective method

Classic method

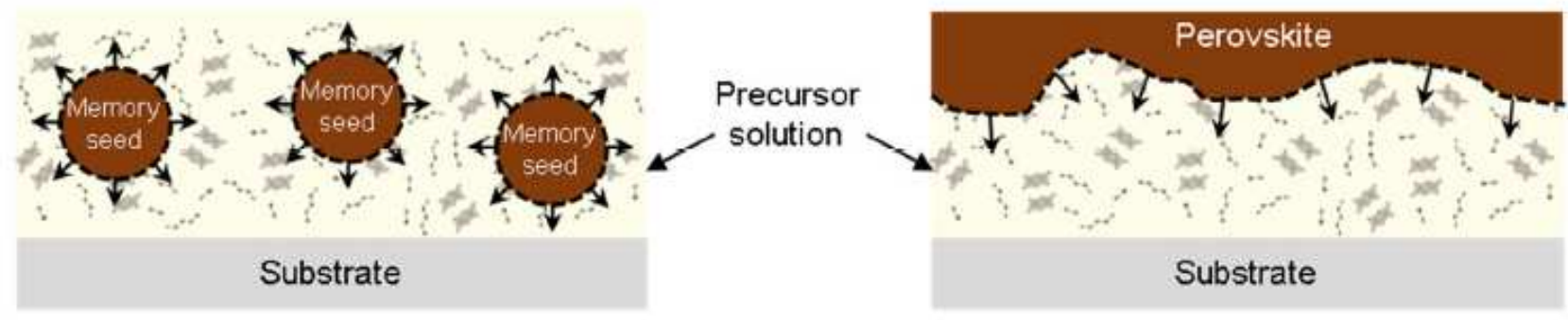

Figure 3 
Nucleation and grain formation from solution to thin films in the Ruddlesden- Popper $n=32 D$ perovskites. $a, b$, In-situ visualization of the formation of grains in 2D perovskite thin films and comparison between the phase-selective synthesis method and the classic one. c, Optical absorbance of the precursor solutions obtained before spin coating and annealing. The gray region corresponds to the absorption of Pbl2. d, Correlation function versus delay time derived from dynamic light scattering performed on the precursor solutions. Dashed lines are fit to the data. Gray and orange regions indicate small and large particle size in solution, respectively. e, Statistical distribution of the particle size in solution and comparison between our phaseselective synthesis method and the classic one. $f$, Schematics of the thin film formation. In the classic method solvated intermediates phases form first such as the onedimensional nonperovskite compounds, which subsequently convert to mixtures of final 2D phases leaving the original crystal shapes of the intermediates as in imprint in the film morphology.
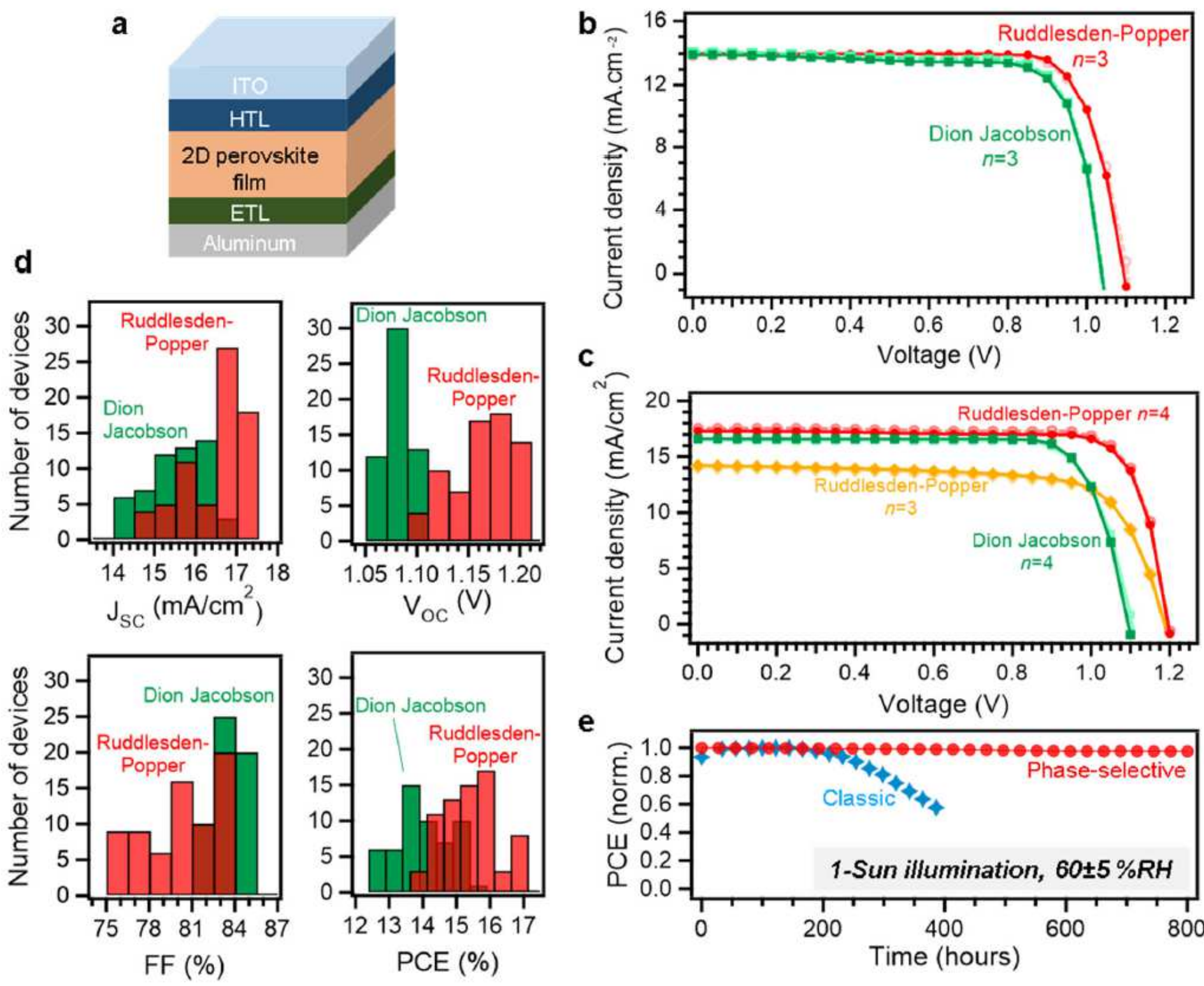

e

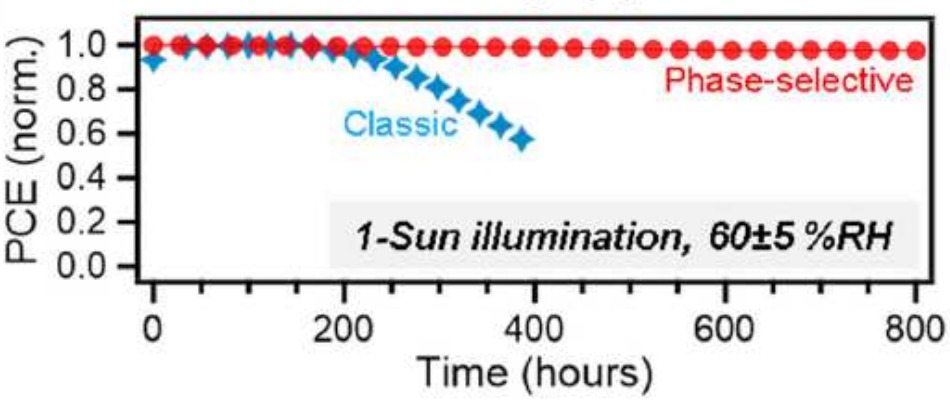

Figure 4 
2D perovskite solar cell performances and stability. a, Schematics of the solar cell devices where HTL and ETL stand for the hole and electron transporting layers. b, Current-voltage characteristics of the cells using the $n=3$ thin films of Ruddlesden-Popper (BA) and Dion Jacobson (3AMP) prepared with the phaseselective method. The HTL is PEDOT:PSS in this case. c, Current-voltage characteristics of our champion solar cells using 2D perovskite thin films of Ruddlesden-Popper (BA) with $n=3$ and $n=4$, and Dion Jacobson (3AMP) with $n=4$ synthetized with the phase-selective method. The HTL is NiOx here. Lightcolored curves correspond to reverse voltage bias scans. d, Statistical distribution of the figures of merit of the $n=42 D$ perovskite solar cells shown in c. e, Stability data for a Ruddlesden-Popper (BA) with $n=4$ solar cell under constant 1-Sun illumination and $60 \% \mathrm{RH}$ compared to the stability measured in a reference thin film solar cell fabricated with the classic method. 